\title{
FOURIER INVERSION FOR UNIPOTENT INVARIANT INTEGRALS
}

\author{
BY \\ DAN BARBASCH ${ }^{1}$
}

\begin{abstract}
Consider $G$ a semisimple Lie group and $\Gamma \subseteq G$ a discrete subgroup such that $\operatorname{vol}(G / \Gamma)<\infty$. An important problem for number theory and representation theory is to find the decomposition of $L^{2}(G / \Gamma)$ into irreducible representations. Some progress in this direction has been made by J. Arthur and G. Warner by using the Selberg trace formula, which expresses the trace of a subrepresentation of $L^{2}(G / \Gamma)$ in terms of certain invariant distributions. In particular, measures supported on orbits of unipotent elements of $G$ occur. In order to obtain information about representations it is necessary to expand these distributions into Fourier components using characters of irreducible unitary representations of $G$. This problem is solved in this paper for real rank $G=1$. In particular, a relationship between the semisimple orbits and the nilpotent ones is made explicit generalizing an earlier result of R. Rao.
\end{abstract}

1. Introduction. Let $G$ be a connected semisimple Lie group with finite center and Lie algebra g. Let $q$ be any element of $G$ and $O(q)$ be the orbit of $q$ under the adjoint action of $G$. Then $O(q)$ carries a $G$-invariant measure which we denote by $T_{q}$. From the results in [11b] it follows that $T_{q}$ has the important property that it defines a tempered distribution on $G$ and is also a measure on $G$. Then it makes sense to try to obtain Fourier inversion formulas in the sense of Harish-Chandra for the distributions $T_{q}$. The problem considered in this paper is to find such explicit formulas. This means to express the distribution $T_{q}$ in terms of a series of tempered invariant eigendistributions of $G$. These series include the characters of the discrete series representations (if $G$ has a compact Cartan subgroup), characters of the unitary principal series and certain eigendistributions that can be interpreted as alternating sums of characters [7].

Such formulas have been obtained in [12] for $q$ semisimple and $G$ of real rank 1 . For $q$ regular and $G$ of rank higher than 1 , formulas are obtained in [6].

In this paper we derive an inversion formula for $q$ unipotent in case $G$ has real rank one. These distributions occur in the Selberg trace formula derived in [10]. The inversion formulas derived here are useful in obtaining

Received by the editors June 24, 1976.

AMS (MOS) subject classifications (1970). Primary 43A30, 22 E30.

${ }^{1}$ Supported by NSF grant MCS 7621044. 
information about the multiplicities of irreducible representations occurring in $L_{0}^{2}(\Gamma \backslash G)$, where $\Gamma$ is a discrete subgroup of $G$ with $\operatorname{vol}(\Gamma \backslash G)<\infty$.

The method used for obtaining the formulas is an extension of some unpublished results of R. Rao [11a]. Let $u$ be a unipotent element, $u=\exp X$ and $\{X, H, Y\}$ be a Lie triple. Then let $\mathfrak{U}=X+Z_{Y}$ where $Z_{Y}=\operatorname{Cent}_{q} Y$. In [11a] it is shown that $\mathfrak{U}$ is a transverse to the adjoint action of $G$ on the Lie algebra g. Since both $X$ and $X-Y$ are elements of $\mathfrak{u}$ and $\cup_{t>0} O(t(X-$ $Y)$ ) contains the orbit of $X$ in its closure, the formula in Theorem 6.7 can be proved for a general class of nilpotents (such that ad $H$ has even eigenvalues only) by lifting the integrals to the transverse $\mathfrak{u}$. The main difficulties in using this method for an arbitrary nilpotent are the fact that $\mathfrak{u}^{G}$ is not equal to the entire Lie algebra $\mathfrak{g}$, the fact that there may be several conjugacy classes of nilpotents conjugate under $G_{c}$ but not under the real group $G$ and finally convergence problems on $\mathfrak{u}$.

In order to avoid these difficulties we take a slightly different approach. In $\S \S 4$ and 5 we construct transverses to the orbits of $X$ and $Z=X-Y$ by using a decomposition theorem of Mostow [9] and lift the invariant measures $T_{X}$ and $T_{Z}$ to the corresponding transverses. Then it becomes apparent that we only need to consider the case su( 2,1$)$. In $\$ 6$ the relation between $T_{Z}$ and $T_{X}$ is calculated. The central result is Theorem 6.7. In $\$ 7$ the Fourier inversion formula for $T_{u}$ is derived by differentiation from the formulas in [12], while in $\S 8$ the constants relevant to the results in [10] are computed explicitly. These results as well as the inversion for an arbitrary $q$ are proved in a different way in [2].

I am indebted to Professor R. Rao for many helpful suggestions.

2. Notation and preliminary results. Let $g$ be a real semisimple Lie algebra with complexification $\mathfrak{g}_{c}$. Let $\sigma$ be the Lie conjugation of $\mathfrak{g}_{c}$ defined by $\mathfrak{g}$. Fix once and for all a Cartan decomposition $\mathfrak{g}=\mathfrak{f}+\mathfrak{p}$ of $\mathfrak{g}$. Then $\mathfrak{f}+\sqrt{-1} \mathfrak{p}$ is a compact real form of $g_{c}$. Denote by $\tau$ the conjugation defined by this real form. Then $\tau$ and $\sigma$ commute and $\tau \sigma$ is a Cartan involution of $g$ giving rise to the Cartan decomposition $\mathfrak{g}=\mathfrak{f}+\mathfrak{p}$. Choose a subspace $a$ and $\mathfrak{n}$ such that $\mathfrak{a}$ is maximal abelian in $\mathfrak{p}$ and $\mathfrak{g}=\mathfrak{f}+\mathfrak{a}+\mathfrak{n}$ is the Iwasawa decomposition of g.

Let $G$ be a connected semisimple Lie group with finite center and Lie algebra $g$. If $G_{c}$ is the connected simply connected group corresponding to $\mathfrak{g}_{c}$, we assume that $G$ is the real subgroup of $G_{c}$ corresponding to $\mathrm{g}$. We also

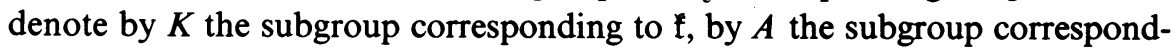
ing to a and by $N$ the subgroup corresponding to $n$.

Let $i \subseteq \mathfrak{g}$ be an Iwasawa Cartan subalgebra which is $\tau$-invariant and has the property that $i \cap \mathfrak{p}=\mathfrak{a}$. Then there is a direct sum decomposition $\mathrm{i}=\mathrm{a}_{K}+\mathfrak{a}$ where $a_{K}=\dot{i} \cap \mathfrak{f}$. If $J$ is the Cartan subgroup with Lie algebra $\dot{i}$, 
then $J=A \cdot A_{K}$ where $A=\exp$ a and $A_{K}=J \cap K$.

Denote by $\Delta\left(\mathfrak{g}_{c}, \dot{\mathrm{l}}_{c}\right)$ the root system of $\mathfrak{g}_{c}$ corresponding to $\dot{\mathrm{l}}_{c}$. We will drop the parentheses and write $\Delta$ whenever there is no danger of confusion. It is well known that if $\beta \in \Delta$ then $\sigma \beta \in \Delta$ where $\sigma \beta$ is defined by $(\sigma \beta)(H)=\overline{\beta(\sigma H)}$ (the bar denotes complex conjugation) for any $H \in \dot{\mathrm{i}}_{c}$.

We also denote by $\Delta(\mathfrak{g}, a)$ the restricted root system and introduce compatible orderings on $\Delta\left(g_{c}, \dot{\mathrm{t}}_{c}\right)$ and $\Delta(\mathrm{g}, \mathrm{a})$ such that if $\beta>0$ then $\sigma \beta>0$ as well, provided $\beta+\sigma \beta \neq 0$. A root such that $\beta+\sigma \beta=0$ is called imaginary and is characterized by the fact that $\left.\beta\right|_{a}=0$. We denote the set of imaginary roots by $\Delta_{0}$. A root $\alpha$ is called real if $\alpha=\sigma \alpha$. It is characterized by the property that $\left.\alpha\right|_{a_{K}}=0$.

Let $B$ be the Cartan-Killing form of $g_{c}$ and $B_{\tau}(X, Y)=B(X, \tau Y)$ be the associated negative definite form. The restriction of $B$ to $\dot{i}_{c}$ will be denoted by $\left(\right.$, ). Since $B$ is nondegenerate we can define a form on the dual of $\dot{i}_{c}$ which will be denoted by ( , ) as well. For $\beta \in \Delta$ define $H_{\beta}$ and $H_{\beta}^{\prime}$ by the relations $\left(H, H_{\beta}\right)=\beta(H)$ for any $H$, and $H_{\beta}^{\prime}=(2 /(\beta, \beta)) H_{\beta}$.

Let $E_{\beta}$ be the root vector associated to the root $\beta$. Then $\sigma E_{\beta}=\rho_{\beta} E_{\sigma \beta}$ where $\left|\rho_{\beta}\right|=1$. We use the Weyl normalization for these vectors so that $\tau E_{\beta}=$ $-E_{-\beta}$. Then the vectors $E_{\beta}, E_{-\beta}$ and $H_{\beta}^{\prime}$ satisfy the relations

$$
\left[H_{\beta}^{\prime}, E_{\beta}\right]=2 E_{\beta}, \quad\left[H_{\beta}^{\prime}, E_{-\beta}\right]=-2 E_{-\beta}, \quad\left[E_{\beta}, E_{-\beta}\right]=-H_{\beta}^{\prime} .
$$

The following well-known facts are going to be used later.

Lemma 2.1. Let $\beta, \gamma \in \Delta$. Then there are integers $p, q \in \mathbf{N}$ such that $\gamma+s \beta$ is a root if and only if $-q \leqslant s \leqslant p$ and $s$ is an integer. In addition $2(\beta, \gamma) /(\gamma, \gamma)=q-p$ and $\beta-2(\beta, \gamma) \gamma /(\gamma, \gamma)$ is a root.

Proof. See [13, p. 41].

Corollary 2.2. Let $\alpha, \beta \in \mathrm{i}_{c}^{*}$, the dual of $\dot{\mathrm{i}}_{c}$. Then

$$
|(\alpha, \beta)|^{2} \leqslant(\alpha, \alpha)(\beta, \beta) .
$$

If $\alpha, \beta \in \Delta$ then equality holds if and only if $\alpha= \pm \beta$.

Proof. The first statement is the Cauchy-Schwartz inequality. The second is a consequence of the fact that the only multiples of $\beta$ that are roots are $\pm \beta$.

LEMMA 2.3. If $\beta$ is a root then $\beta-\sigma \beta$ is not a root.

Proof. See [1, p. 6].

LEMMA 2.4. Let $\beta$ and $\gamma$ be positive roots such that $\beta \neq \gamma$ and $\beta \neq \sigma \gamma$. If $\left.\beta\right|_{a}$ and $\left.\gamma\right|_{\mathfrak{a}}$ are multiples of each other then either $\beta-\gamma$ or $\beta-\sigma \gamma$ is a root. 
Proof. We have the relation

$$
(\beta, \gamma)+(\beta, \sigma \gamma)=(\beta, \gamma+\sigma \gamma)=\left(\beta,\left.2 \gamma\right|_{a}\right)=2\left(\left.\beta\right|_{a},\left.\gamma\right|_{a}\right)>0,
$$

so either $(\beta, \gamma)>0$ or $(\beta, \sigma \gamma)>0$, say $(\beta, \gamma)>0$. Then Lemma 2.1 implies that $\beta-\gamma$ must be a root and the proof is complete.

Next, we consider the conjugacy classes of Cartan subalgebras of $g$ for the case when $\operatorname{rank}(G / K)=1$. It is well known that any Cartan subalgebra can be conjugated to a $\tau$-invariant one. Let $\mathfrak{h}$ be such a subalgebra and $\mathfrak{h}=\mathfrak{h}_{k}+$ $\mathfrak{h}_{p}$ be its Cartan decomposition. Since $\operatorname{rank}(G / K)=1$ it follows that $\operatorname{dim} \mathfrak{h}_{p}$ $\leqslant 1$. The Cartan subalgebras such that $\operatorname{dim} \mathfrak{h}_{p}=1$ are all conjugate to the Iwasawa Cartan subalgebra $\dot{i}$. The Cartan subalgebras such that $\operatorname{dim} \mathfrak{h}_{p}=0$ are all conjugate. They are called fundamental or also compact Cartan subalgebras. The following result is well known. We include a proof for completeness.

PROPOSITION 2.5. Let $\mathrm{g}$ be a real semisimple algebra such that $\operatorname{rank}(G / K)=$ 1. Then we have the following two cases.

(1) If rank $g>$ rank then the fundamental and the Iwasawa Cartan subalgebra are the same. There is only one conjugacy class of Cartan subalgebras.

(2) If rank $g=$ rank then there is a real root $\alpha \in \Delta\left(g_{c}, j_{c}\right)$. A representative for the fundamental Cartan subalgebra is $\mathfrak{b}=\mathfrak{a}_{K}+\mathbf{R}\left(E_{\alpha}-E_{-\alpha}\right)$. The subalgebras $\mathrm{i}$ and $\mathfrak{b}$ are not conjugate by $G$ but the element $\nu=\exp \left[\pi \sqrt{-1}\left(E_{\alpha}+\right.\right.$ $\left.E_{-\alpha}\right) / 4$ ] conjugates $\dot{i}_{c}$ into $\mathfrak{b}_{c}$.

Proof. The first statement is straightforward. Assume rank $g=$ rank $f$. Let $\mathfrak{b} \subseteq \mathfrak{f}$ be a Cartan subalgebra. Since not all roots of $\Delta\left(\mathfrak{g}_{c}, \mathfrak{b}_{c}\right)$ are compact, there is a singular imaginary root $\tilde{\alpha}$. Select $Z \in \mathfrak{b}$ such that the only roots vanishing at $Z$ are $\pm \tilde{\alpha}$. Let $c_{Z}$ be the center of $\operatorname{Cent}_{\mathrm{g}} Z$ and $l_{Z}$ be the derived algebra. Then $l_{Z}$ is isomorphic to $\mathrm{sl}(2, \mathbf{R})$ and we can select a basis $X^{*}, H^{*}$, $Y^{*}$ such that $\tau X^{*}=-Y^{*}$ and $\left[X^{*}, Y^{*}\right]=H^{*},\left[H^{*}, X^{*}\right]=2 X^{*},\left[H^{*}, Y^{*}\right]=$ $-2 Y^{*}$. Then $\mathfrak{b}=c_{Z}+\mathbf{R}\left(X^{*}-Y^{*}\right)$. Define $\mathfrak{h}=\mathbf{R} H^{*}+c_{Z}$. Then $\mathfrak{h}$ is an Iwasawa Cartan subalgebra and if $\mu=\exp \left[\sqrt{-1} \pi\left(X^{*}+Y^{*}\right) / 4\right]$ then $\mathfrak{h}_{c}^{\mu}=$ $\mathfrak{b}_{c}$. Furthermore $\tilde{\alpha}^{\mu}=\tilde{\alpha} \circ \mu$ is a real root of $\Delta\left(\mathfrak{g}_{c}, \mathfrak{h}_{c}\right)$ since $\mu$ leaves $c_{Z}$ invariant and $\tilde{\alpha}$ vanishes on $c_{Z}$. The Cartan subalgebra $\mathfrak{h}$ is conjugate to $\dot{j}$ by some element in $G$.

Conversely, let $\alpha \in \Delta\left(\mathrm{g}_{c}, \dot{\mathrm{i}}_{c}\right)$ be a real root and $E_{ \pm \alpha}$ be the corresponding root vectors. Since $\left|\rho_{\alpha}\right|=1$ we can choose $t_{\alpha} \in \mathbf{R}$ such that $\exp \left[2 \sqrt{-1} t_{\alpha}\right]=$ $\rho_{\alpha}$. Let $h_{\alpha}=\exp \left[\sqrt{-1} t_{\alpha} H_{\alpha}^{\prime}\right.$. Then $\tau\left(h_{\alpha}\right)=h_{\alpha}$. If we replace the root vectors $E_{\beta}$ by $\operatorname{Ad} h_{\alpha}\left(E_{\beta}\right)$ the Weyl normalization is preserved. In addition

$$
\begin{aligned}
\sigma\left(\operatorname{Ad} h_{\alpha} E_{\alpha}\right) & =\sigma\left(\exp \left(\sqrt{-1} t_{\alpha}\right) E_{\alpha}\right)=\exp \left(-\sqrt{-1} t_{\alpha}\right) \rho_{\alpha} E_{\alpha} \\
& =\exp \left(\sqrt{-1} t_{\alpha}\right) E_{\alpha}=\operatorname{Ad} h_{\alpha} E_{\alpha} .
\end{aligned}
$$


Similarly $\sigma\left(\operatorname{Ad} h_{\alpha} E_{-\alpha}\right)=\operatorname{Ad} h_{\alpha} E_{-\alpha}$ so $E_{ \pm \alpha} \in \mathfrak{g}$. Then

$$
\mathfrak{b}=\mathbf{R}\left(E_{\alpha}-E_{-\alpha}\right)+\mathfrak{a}_{K}
$$

is a compact Cartan subalgebra. Finally the fact that $\operatorname{Ad} \nu$ maps $\mathfrak{b}_{c}$ into $\dot{i}_{c}$ follows from an elementary calculation in $\mathrm{sl}(2, \mathrm{C})$. This completes the proof.

From here on $\alpha$ will always denote a positive real root. Fix a positive restricted root $\lambda \in \Delta(\mathfrak{g}, \mathfrak{a})$ such that the only multiples of $\lambda$ that are also roots are $\pm \lambda$ and possibly $\pm \frac{1}{2} \lambda$. Let

$$
\begin{aligned}
\Delta_{0} & =\left\{\beta \in \Delta\left(g_{c}, \dot{\mathrm{l}}_{c}\right):\left.\beta\right|_{a}=0\right\}, \\
P_{1} & =\left\{\beta \in \Delta\left(\mathfrak{g}_{c}, \dot{\mathrm{l}}_{c}\right):\left.\beta\right|_{a}=\frac{1}{2} \lambda\right\}, \\
P_{2} & =\left\{\beta \in \Delta\left(\mathrm{g}_{c}, \dot{\mathrm{l}}_{c}\right):\left.\beta\right|_{a}=\lambda\right\}, \\
\Psi & =\left\{\gamma \in \Delta_{0}: \alpha \pm \gamma \text { are not roots }\right\} .
\end{aligned}
$$

Lemma 2.6. If $P_{1} \neq \varnothing$ then there is a real root $\alpha \in \Delta\left(\mathfrak{g}_{c}, \dot{i}_{c}\right)$ such that $\left.\alpha\right|_{a}=\lambda$. Also $H_{\lambda}^{\prime}=H_{\alpha}^{\prime}$ and ad $H_{\lambda}^{\prime}$ has eigenvalues $0, \pm 1$ and \pm 2 .

Proof. Let $\beta \in P_{2}$ and denote by $\alpha$ the functional on $i$ which equals $\lambda$ on $a$ and 0 on $a_{K}$. We have to show that $\alpha \in \Delta$. Assume not. Then for any $\alpha_{1} \in P_{1}$, the sum $\alpha_{1}+\sigma \alpha_{1} \notin \Delta$. Since $\alpha_{1}-\sigma \alpha_{1} \notin \Delta$ by Lemma 2.3, it follows that $\left(\alpha_{1}, \sigma \alpha_{1}\right)=0$. Since $\alpha_{1}+\sigma \alpha_{1}=\alpha$ we also get $2\left(\alpha_{1}, \alpha_{1}\right)=(\alpha, \alpha)$. On the other hand

$$
\frac{2\left(\beta, \alpha_{1}\right)}{\left(\alpha_{1}, \alpha_{1}\right)}+\frac{2\left(\beta, \sigma \alpha_{1}\right)}{\left(\sigma \alpha_{1}, \sigma \alpha_{1}\right)}=\frac{2(\beta, \alpha)}{\left(\alpha_{1}, \alpha_{1}\right)}=\frac{2(\alpha, \alpha)}{\frac{1}{2}(\alpha, \alpha)}=4 .
$$

At least one of the summands must be larger than 2 , say $2\left(\beta, \alpha_{1}\right) /\left(\alpha_{1}, \alpha_{1}\right) \geqslant$ 2. Since $\beta$ and $\alpha_{1}$ cannot be multiples of each other, Lemma 2.1 implies that $\beta-\alpha_{1}$ is a root. Corollary 2.2 implies that

$$
\frac{2\left(\beta, \alpha_{1}\right)}{\left(\alpha_{1}, \alpha_{1}\right)} \cdot \frac{2\left(\beta, \alpha_{1}\right)}{(\beta, \beta)}<4
$$

and since both numbers are integers, $2\left(\beta, \alpha_{1}\right) /\left(\alpha_{1}, \alpha_{1}\right)<4$. Together with (1) this implies $\left(\beta, \sigma \alpha_{1}\right)>0$ so $\beta-\sigma \alpha_{1}$ is also a root. Both $\beta-\alpha_{1}$ and $\beta-\sigma \alpha_{1}$ restrict to $\frac{1}{2} \lambda$ and they cannot be multiples of each other. Thus we can apply Lemma 2.4 to conclude that either $\beta-\alpha_{1}-\left(\beta-\sigma \alpha_{1}\right)=\sigma \alpha_{1}-\alpha_{1}$ or $\beta-$ $\alpha_{1}-\left(\sigma \beta-\alpha_{1}\right)=\beta-\sigma \beta$ is a root. This contradicts the statement of Lemma 2.3. Thus $\alpha \in \Delta$. Since $\left.\alpha\right|_{a_{K}}=0$ it follows that $H_{\alpha}^{\prime}=H_{\lambda}^{\prime}$ and $\beta\left(H_{\lambda}^{\prime}\right)=2$, $\alpha_{1}\left(H_{\lambda}^{\prime}\right)=1$. This completes the proof.

Let $B$ be the Cartan subgroup corresponding to $\mathfrak{b}$. We normalize the measures on $G, \mathfrak{g}, J, \mathrm{i}, B$ and $\mathfrak{b}$ as in [15a, \$8.1.2]. Furthermore we normalize the Haar measures on any compact subgroup so that it has volume one. 
Let $\mathfrak{b}^{*}$ denote the dual to $\sqrt{-1} \mathfrak{b}$. Then we define a lattice $L_{B}$ in $\mathfrak{b}_{c}^{*}$ by

$$
L_{B}=\left\{\mu \in \mathfrak{b}_{c}^{*}: \frac{2(\mu, \beta)}{(\beta, \beta)} \in \mathbf{Z} \text { for any } \beta \in \Delta\left(\mathfrak{g}_{c}, \mathfrak{b}_{c}\right)\right\} \text {. }
$$

Then the element $\rho=\frac{1}{2} \Sigma_{\beta>0} \beta$ is in this lattice. For any $\mu \in L_{B}$ we can define a character of $B$ by the formula

$$
\xi_{\mu}(\exp H)=\exp \mu\left(H^{\prime}\right) \quad \text { all } H \in \mathfrak{b} .
$$

Let

and

$$
B^{\prime}=\left\{h \in B: \xi_{\beta}(h) \neq 1 \text { for any } \beta \in \Delta\left(\mathfrak{g}_{c}, \mathfrak{b}_{c}\right)\right\}
$$

$$
\Delta_{B}(h)=\xi_{-\rho}(h) \prod_{\beta>0}\left(1-\xi_{\beta}(h)\right) \text { for } h \in B^{\prime} .
$$

For any $f \in C_{c}^{\infty}(G)$ we can define a function $\phi_{f}^{B}$ by

$$
\Phi_{f}^{B}(h)=\Delta_{B}(h) \int_{G / B} f\left(h^{x}\right) d x, \quad h \in B^{\prime} .
$$

This function has the following properties.

(a) $\Phi_{f}^{B} \in \mathcal{S}\left(B^{\prime}\right)$, the Schwartz space of $B^{\prime}$,

(b) if $W(G, B)$ denotes the Weyl group of $G$ with respect to $B$ then $\Phi_{f}^{B}(w h)=(\operatorname{det} w) \Phi_{f}^{B}(h)$ for any $w \in W(G, B)$.

The function $\Phi_{f}^{B}$ plays an important role in harmonic analysis on semisimple groups. In particular the following result will be useful later.

LEMma 2.7. Let $\gamma$ be an element of $B$. Let $P_{\gamma}=\left\{\beta \in \Delta\left(\mathfrak{g}_{c}, \mathfrak{b}_{c}\right)\right.$ such that $\xi_{\beta}(\gamma)=1$ and $\left.\beta>0\right\}$ and $P_{g / \gamma}=\left\{\beta>0: \beta \notin P_{\gamma}\right\}$. Define a differential operator $\bar{\omega}_{\gamma}$ by $\bar{\omega}_{\gamma}=\prod_{\beta \in P_{\gamma}} H_{\beta}$. Then

$$
\lim _{\substack{\tilde{\gamma} \rightarrow \gamma \\ \tilde{\gamma} \in B^{\prime}}} \Phi_{f}^{B}\left(\tilde{\gamma} ; \bar{\omega}_{\gamma}\right)=k_{\gamma} \int_{G / G_{\gamma}} f\left(\gamma^{\dot{x}}\right) d \dot{x}
$$

where $k_{\gamma}=d_{\gamma} \xi_{-\rho}(\gamma) \Pi_{\beta \in P_{g / \gamma}}\left(\xi_{\beta}(\gamma)-1\right)$ and $d_{\gamma}$ depends only on the measure $d \dot{x}$.

Proof. See [4d, p. 33].

To each element $\mu \in L_{B}$ there is, according to [4c, p. 281], associated an invariant central eigendistribution $\Theta_{\mu}$ which is a locally summable function on $G$ and analytic on the set of regular points. If $\Theta_{\mu}$ is regular i.e. $w \mu \neq \mu$ for any $w \in W\left(G_{c}, B_{c}\right)$ then $\Theta_{\mu}$ is the character of a discrete series representation of $G$. For the explicit form of $\Theta_{\mu}$ for real rank 1 see [12].

Let $W_{I}$ be the Weyl group corresponding to the imaginary roots of $\mathrm{i}$. Then $W_{I}$ can be identified with the Weyl group $W\left(M, A_{K}\right)$ where $M=$ Cent $_{K} A$. A 
unitary character $\chi \in \hat{A}_{K}$ is called regular if $w \chi \neq \chi$ for any $w \in W_{I}$. To each pair $(\chi, \nu)$ where $\chi \in \hat{A}_{K}$ is regular and $\nu \in \hat{A} \approx \mathbf{R}$ there is associated a principal series character which we denote by $T^{(\chi, \nu)}$.

Let $q \in G$ be an arbitrary element. According to [3, p. 235], the group $G_{q}=\operatorname{Cent}_{G} q$ is unimodular so that the homogeneous space $G / G_{q}$ carries an invariant measure. In [11b] it was proved that the integral

$$
T_{q}(f)=\int_{G / G_{q}} f\left(q^{\dot{x}}\right) d \dot{x}
$$

is convergent for any $f \in C_{c}(G)$. In particular, this implies that $T_{q}$ is a tempered invariant distribution on $G$. We normalize the measures $d x^{*}$ on $G / G_{q}$ and $d y$ on $G_{q}$ so that

$$
\int_{G} f(x) d x=\int_{G / G_{q}} \int_{G_{q}} f\left(x^{*} y\right) d x^{*} d y
$$

for any $f \in C_{c}(G)$.

3. Conjugacy classes of nilpotent elements. An element $X \in \mathfrak{g}$ is called nilpotent if ad $X$ is a nilpotent transformation of $\mathrm{g}$. A triple $\{X, H, Y\}$ is called a Lie triple if the following relations hold:

$$
[H, X]=2 X, \quad[H, Y]=2 Y \text { and }[X, Y]=-H .
$$

Then the real algebra generated by $X, H$ and $Y$ is isomorphic to sl(2, R). The Jacobson-Morozov theorem [8a] implies that any nilpotent element $X$ in a complex semisimple Lie algebra $g_{c}$ can be embedded in a Lie triple $\{X, H, Y\}$. If in addition $X \in \mathrm{g}$ then $H$ and $Y$ can be chosen to be in $\mathrm{g}$ as well.

Two Lie triples $\{X, H, Y\}$ and $\left\{X_{1}, H_{1}, Y_{1}\right\}$ are said to be conjugate by $G$ or real conjugate if there is $x \in G$ such that $X^{x}=X_{1}, Y^{x}=Y_{1}$ and $H^{x}=$ $H_{1}$.

The conjugacy classes of Lie triples and nilpotent elements are characterized in the next proposition.

Proposition 3.1. Two Lie triples, $\{X, H, Y\}$ and $\left\{X_{1}, H_{1}, Y_{1}\right\}$, are conjugate if and only if their nilpotent components $X$ and $X_{1}$ are conjugate. The Lie triples are conjugate by $G_{c}$ if and only if $H$ and $H_{1}$ are conjugate by $G_{c}$. The Lie triples are conjugate by $G$ if and only if $Z=X-Y$ and $Z_{1}=X_{1}-Y_{1}$ are conjugate.

Proof. The proof of the first two statements is contained in [8a]. The last statement was formulated by R. Rao and proved by B. Kostant based on [8b]. Since no proof seems to have appeared in print, we give one for completeness.

Assume that $X_{1}$ and $X$ are conjugate by $G$. Then by the previous statement, 
there is $x \in G$ such that $X_{1}^{x}=X, Y_{1}^{x}=Y$ and $H_{1}^{x}=H$. Thus $\left(X_{1}-Y_{1}\right)^{x}=$ $X-Y$. Conversely, assume that there is $x \in G$ such that $\left(X_{1}-Y_{1}\right)^{x}=X-$ $Y$. By Theorem 6 in [9] we may assume that the two Lie triples are normalized so that $\theta X=-Y$ and $\theta X_{1}=-Y_{1}$. Then $X-Y$ and $X_{1}-Y_{1}$ are in $\ell$ and in addition we may assume that $x \in K$. We then replace the Lie triples $X_{1}$, $H_{1}, Y_{1}$ by $X_{1}^{x}, H_{1}^{x}, Y_{1}^{x}$ and call it $X_{1}, H_{1}, Y_{1}$ once again. Thus we assume $X_{1}-Y_{1}=X-Y$. Since $X-Y$ is conjugate by $\operatorname{SL}(2, \mathrm{C})$ to $\sqrt{-1} H$ we replace the Lie triples by the Lie triples

$$
\left\{\frac{1}{2}(X+Y+i H), i(X-Y), \frac{1}{2}(X+Y-i H)\right\}
$$

and

$$
\left\{\frac{1}{2}\left(X_{1}+Y_{1}+i H_{1}\right), i\left(X_{1}-Y_{1}\right), \frac{1}{2}\left(X_{1}+Y_{1}-i H_{1}\right)\right\} \text {. }
$$

Let $\mathfrak{f}_{c}$ and $\mathfrak{p}_{c}$ be the complexifications of $\mathfrak{f}$ and $\mathfrak{p}$. Furthermore let $\tau_{c}$ be the complex linear map defined by $\left.\tau_{c}\right|_{\mathfrak{t}_{c}}=$ id and $\left.\tau_{c}\right|_{p_{c}}=-$ id. We then note that, due to the assumptions on $G_{c}$, the map $\tau_{c}$ extends to a map on $G_{c}$. Let $K_{\tau}$ be the set of fixed points of $\tau_{c}$ and $\tilde{K}_{\tau}$ the image of $K_{\tau}$ by the adjoint map. If $\tilde{K}$ is the connected component of $\tilde{K}_{\tau}$ then by Proposition 1 in [8b] we can write $\tilde{K}_{\tau}=F \cdot \tilde{K}$ where $F$ is the set of elements of order two in exp ad $a_{c}$. Also, $\tilde{K}_{\tau}$ is the set of fixed points of $\tau_{c}$ in the adjoint group of $\mathfrak{g}_{c}$. In the terminology of [8b] the two Lie triples are called normal. Due to the remark following Proposition 4 in [8b] the two Lie triples are conjugate by some element $\tilde{x} \in \tilde{K}_{\tau}$. We note that $\tilde{K}=\operatorname{Ad} K \exp \sqrt{-1}$ ad f. Since $K \subseteq G$ is connected we may assume $\tilde{x}=f \exp \sqrt{-1}$ ad $Z$ where $Z \in \mathfrak{f}$. Since $(X-Y)^{\tilde{x}}=X-$ $Y$ we also have $\tau\left\{(X-Y)^{\tilde{x}}\right\}=\tau(X-Y)$ so $(X-Y)^{\tau(\tilde{x})}=X-Y$. But $\tau(\tilde{x})=f \exp (-\sqrt{-1}$ ad $Z)$ so $\tilde{x}^{-1} \tau(\tilde{x})=\exp 2 \sqrt{-1}$ ad $Z$ also centralizes $X-Y$. But then

$$
\begin{aligned}
X-Y= & \exp 2 \sqrt{-1} \text { ad } Z(X-Y)=\cosh (2 \sqrt{-1} \text { ad } Z)(X-Y) \\
& +\sinh (2 \sqrt{-1} \text { ad } Z)(X-Y) .
\end{aligned}
$$

Since the first term on the right-hand side is in $f$ and the second in $\sqrt{-1}$ it follows that

$$
\sinh (2 \sqrt{-1} \text { ad } Z)(X-Y)=0 .
$$

But $2 \sqrt{-1}$ ad $Z$ has real eigenvalues only, so ad $Z(X-Y)=0$. This also implies $f \cdot(X-Y)=X-Y$ so $f$ leaves the entire Lie triple fixed. Thus we also get $\exp (\sqrt{-1}$ ad $Z) H=H_{1}$. Again

$$
H_{1}=\exp (\sqrt{-1} \text { ad } Z) H=\cosh (\sqrt{-1} \text { ad } Z) H+\sinh (\sqrt{-1} \text { ad } Z) H
$$

so $\sinh (\sqrt{-1}$ ad $Z) H=0$ since it is contained in $\sqrt{-1} \mathfrak{p}$ (while the other term is in $\mathfrak{p})$. But this implies $(\operatorname{ad} Z) H=0$ so $H=H_{1}$. Therefore the two Lie triples must coincide and the proof is complete. 
We now determine the conjugacy classes of nilpotent elements in a semisimple Lie algebra of real rank one. Let $\lambda$ be the restriction of the real positive root $\alpha$ to $a$ or in the absence of a real root let $\lambda$ be the restriction of a simple positive root of $\Delta\left(\mathfrak{g}_{c}, \dot{d}_{c}\right)$. Define $H_{\lambda}^{\prime}=2 H_{\lambda} /(\lambda, \lambda)$ as in $\$ 2$. Let $g_{i}=\left\{Z \in g:\left[H_{\lambda}^{\prime}, Z\right]=i Z\right\}$.

THEOREM 3.2. The real conjugacy classes of nilpotent elements are given by

(1) $\left\{X, H_{\lambda}^{\prime}, Y\right\}$ where $X \in g_{2}$ and $\left\{-X, H_{\lambda}^{\prime},-Y\right\}$ when $\operatorname{dim} g_{2}=1$,

(2) $\left\{X_{1}, 2 H_{\lambda}^{\prime}, Y_{1}\right\}$ where $X_{1} \in g_{1}$.

Proof. Let $\{X, H, Y\}$ be an arbitrary Lie triple. Since $X, H$ and $Y$ generate a semisimple algebra isomorphic to $\mathrm{sl}(2, \mathbf{R})$, ad $H$ must have integral eigenvalues only. Furthermore Theorem 6 in [9] implies that we can normalize the Lie triple so that $\tau X=-Y$ and $\tau H=-H$. Since $H \in \mathfrak{p}$ we can conjugate the Lie triple by an element in $K$ so that $H=\varepsilon H^{\prime}$ where $\varepsilon>0$. We note that ad $H_{\lambda}^{\prime}$ has eigenvalues $0, \pm 2$ and possibly \pm 1 . On the other hand, ad $H$ has an eigenvalue equal to +2 and thus we can only have $\varepsilon=1$ or $\varepsilon=2$. Furthermore, if $\varepsilon=2$ then ad $H_{\lambda}^{\prime}$ has to have eigenvalue +1 so in the notation of $\S 2$ we have $P_{1} \neq \varnothing$. We thus have two types of Lie triples.

(1) $\left\{X_{1}, 2 H_{\lambda}^{\prime}, Y_{1}\right\}$ where $X_{1} \in g_{1}$,

(2) $\left\{X, H_{\lambda}^{\prime}, Y\right\}$ where $X \in g_{2}$.

Due to Proposition 3.1 the two types are in different conjugacy classes. By using Theorem 8.11.3 in [14] we conclude that the Lie triples of type (1) are one conjugacy class and the Lie triples of type (2) are one conjugacy class when $\operatorname{dim} g_{2}>1$. It remains to show that if $\operatorname{dim} g_{2}=1$ then $\{X, H, Y\}$ is not conjugate to $\{-X, H,-Y\}$. Suppose that there is $x \in G$ such that $X^{x}=-X, H^{x}=H$ and $Y^{x}=-Y$. Then $x \in M=$ Cent $_{K} H$. Let $m$ be the Lie algebra of $M$. Then $[\mathrm{m}, X]=0$, for otherwise $\operatorname{dim} g_{2}>1$. This implies that $M_{0}$, the connected component of $M$, centralizes $X$. Let $G_{c}(H)$ be the centralizer of $H$ in $G_{c}$ and $g_{c}(H)$ its Lie algebra. Since $G_{c}(H)$ is the centralizer of a torus, it is connected. Its Lie algebra has Cartan decomposition

$$
g_{c}(H)=(\mathfrak{m}+\sqrt{-1} \mathbf{R} H)+(\sqrt{-1} \mathfrak{m}+\mathbf{R} H) .
$$

Thus

$$
G_{c}(H)=M \exp \{\sqrt{-1} \mathbf{R} H\} \exp \{\sqrt{-1} \mathrm{~m}+\mathbf{R} H\} .
$$

Thus $M=M_{0} \exp \{\sqrt{-1} \mathbf{R} H\} \cap K$. Suppose $h=\exp \{\sqrt{-1} t H\} \in K$ for some $t \in \mathbf{R}$. Then $\sigma(\exp \{\sqrt{-1} t H\})=\exp \{\sqrt{-1} t H\}$ so $\exp \{\sqrt{-1} t H\}=\exp \{-\sqrt{-1} t H\}$ or $\exp \{2 \sqrt{-1} t H\}=e$. Since $H$ as an element of $g_{c}$ is contained in a subalgebra isomorphic to $\mathrm{sl}(2, \mathbf{C})$ it follows that $t=k \pi$ for some $k \in \mathbf{Z}$. But then

$$
X^{h}=\exp \{2 \sqrt{-1} t\} X=\exp \{2 \sqrt{-1} k \pi\} X=X .
$$


Thus there cannot be such $x \in M$ and the proof is complete.

EXAMPLE. Let $g=\operatorname{su}(2,1)$. Then representatives of all conjugacy classes are given by

$$
\begin{gathered}
X=\left[\begin{array}{ccc}
0, & 0, & 0 \\
0, & i / 2, & i / 2 \\
0, & -i / 2, & -i / 2
\end{array}\right], \quad H_{\lambda}^{\prime}=\left[\begin{array}{ccc}
0, & 0, & 0 \\
0, & 0, & -1 \\
0, & -1, & 0
\end{array}\right], \\
Y=\left[\begin{array}{ccc}
0, & 0, & 0 \\
0, & i / 2, & -i / 2 \\
0, & i / 2, & -i / 2
\end{array}\right] .
\end{gathered}
$$

In this case $X$ and $-X$ are not conjugate.

$$
\begin{gathered}
X=\left[\begin{array}{ccc}
0, & 1, & 1 \\
-1, & 0, & 0 \\
1, & 0, & 0
\end{array}\right], \quad 2 H_{\lambda}^{\prime}=\left[\begin{array}{ccc}
0, & 0, & 0 \\
0, & 0, & -2 \\
0, & -2, & 0
\end{array}\right], \\
Y=\left[\begin{array}{ccc}
0, & 1, & -1 \\
-1, & 0, & 0 \\
-1, & 0, & 0
\end{array}\right] .
\end{gathered}
$$

Remark 3.3. In the case when $P_{1} \neq \varnothing$ let $\alpha_{1} \in P_{1}$. Then the roots $\alpha_{1}, \sigma \alpha_{1}$ and $\alpha$ generate a subalgebra isomorphic to $\operatorname{su}(2,1)$. Thus we can select representatives for the conjugacy classes of nilpotents to be as in the example above. If $P_{1}=\varnothing$ then the representatives can be chosen in $\mathrm{sl}(2, \mathbf{R}) \subseteq \operatorname{su}(2,1)$ to have the same expression as $X, H$ and $Y$ in part (1) of the example.

4. The orbit of the semisimple element $Z$. With the notation as before let $\{X, H, Y\}$ be a Lie triple normalized as before and let $Z_{0}=X-Y$. Let $G_{Z}$ be the centralizer of $Z_{0}$ in $G$ and $g_{Z}$ its Lie algebra. Since $Z_{0}$ is contained in $Z^{\prime}$, $G_{Z}$ is connected and has a Cartan decomposition $G_{Z}=K_{Z} S_{Z}$. Let $g_{Z}=\mathfrak{f}_{Z}+$ $\mathfrak{p}_{Z}$ be the corresponding decomposition for the Lie algebra. Let $L=$ Cent $_{G}\{X, H, Y\}$ and $\mathcal{L}$ be its Lie algebra. If $g_{i}=\{Z:$ ad $H Z=i Z\}$ then let $g_{\mathrm{ev}}=\sum_{i \text { even }} g_{i}$ and $g_{\mathrm{o}}=\sum_{i \text { odd }} g_{i}$. Then $g_{\mathrm{ev}}$ is a $\tau$-invariant subalgebra and therefore reductive. Let $g_{\mathrm{ev}}=\mathfrak{f}_{\mathrm{ev}}+\mathfrak{p}_{\mathrm{ev}}$ be the Cartan decomposition and $\mathfrak{p}_{\mathrm{o}}$ be the orthogonal complement of $\mathfrak{p}_{\mathrm{ev}}$ in $\mathfrak{p}$ with respect to the Cartan-Killing form.

LEMMA 4.1. Let $(\pi, V)$ be an irreducible finite dimensional representation of $\mathrm{sl}(2, \mathrm{C})$ of highest weight $n$. Let $V^{\mathrm{o}}=$ Cent $Z_{0}$ and $V_{i}=\{v: \pi(H) v=i v\}$. Then $V^{\mathrm{o}}=\{0\}$ if $n$ is odd and $V^{\mathrm{o}}$ is isomorphic to $V_{n}$ when $n$ is even.

Proof. Let $v=v_{n}+v_{n-2}+\cdots+v_{-n}$ be an element of $V^{\circ}$. From representation theory of $\operatorname{sl}(2, \mathrm{C})$ e.g. [13, Lemma $\mathrm{A}$ and Theorem B, p. 28] we know that $\operatorname{dim} V_{i}=1$ for $-n \leqslant i \leqslant n, i \equiv n(\bmod 2)$, and $V_{i}=\{0\}$ other- 
wise. Furthermore, $\pi(X) V_{n}=\pi(Y) V_{-n}=\{0\}$ and $\pi(X), \pi(Y)$ are isomorphisms from $V_{i}$ to $V_{i+2}$ and $V_{i}$ to $V_{i-2}$ respectively. The equation $\pi\left(Z_{0}\right) v=0$ decomposes according to eigenspaces of $\pi(H)$ in the following way. Let $i$ be an eigenvalue of $\pi(H)$. Then the component of $\pi\left(Z_{0}\right) v$ in $V_{i}$ gives the equation

$$
\pi(X) v_{i-2}-\pi(Y) v_{i+2}=0 .
$$

Let $j$ be the largest integer so that $v_{j} \neq 0$. For $V_{j+2}$ we get the equation $\pi(X) v_{j}=0$. Thus $j=n$ if $v \neq 0$. Furthermore, for $V_{n}$ we also get $\pi(X) v_{n-2}$ $=0$ so $v_{n-2}=0$. Equation (*) implies $v_{k}=0$ if $n-k \neq 0(\bmod 4)$. Let $k$ be the integer such that $v_{n-4 k} \neq 0$ but $v_{n-4 k-4}=0$. Then $\pi(X) v_{n-4 k-4}-$ $\pi(Y) v_{n-4 k}=0$ so $\pi(Y) v_{n-4 k}=0$. Thus $n-4 k=-n$ or $n=2 k$. This proves the lemma.

COROllaRY 4.2. The linear subspace $\mathbf{R} H+\left[H, \mathfrak{f}_{Z}\right]$ is orthogonal to $\mathfrak{p}_{Z}$. Let $\mathfrak{p}_{1}$ be the orthogonal complement of $\mathbf{R} H+\left[H, \mathfrak{f}_{Z}\right]+\mathfrak{p}_{Z}$ in $\mathfrak{p}$ and $W \in \mathfrak{p}_{1}$, $W \neq 0$. Then $(\mathbf{R} W)^{L}=\mathfrak{p}_{1}$.

Proof. Let $Z_{1} \in \mathfrak{f}_{Z}$ and $Z_{2} \in \mathfrak{p}_{Z}$. Then $B_{\tau}\left(\left[H, Z_{1}\right], Z_{2}\right)=B_{\tau}\left(H,\left[Z_{1}, Z_{2}\right]\right)$. Then $\left[Z_{1}, Z_{2}\right] \in \mathrm{g}_{Z}$. Let $Z \in \mathrm{g}_{Z}$ be arbitrary. Then

$$
B_{\tau}(H, Z)=B([X, Y], Z)=B(Y,[X, Z])=B(Y,[Y, Z])=0
$$

since $\left[Z_{0}, Z\right]=0$ which is the same as $[X, Z]=[Y, Z]$. Thus $\left[H, \mathfrak{t}_{Z}\right]$ is orthogonal to $\mathfrak{p}_{Z}$ and also $H$ is orthogonal to $g_{Z}$. Thus the first statement in the corollary is proved. For the second we note that since $L \subseteq K$ and $\mathbf{R} H+\left[H, \mathfrak{f}_{Z}\right]+\mathfrak{p}_{Z}$ is $L$-invariant, $\mathfrak{p}_{1}$ is also $L$-invariant. Next we show that

$$
\left[H, \mathfrak{f}_{Z}\right]+\mathfrak{p}_{Z}=\left\{Z-\tau Z: Z \in(\operatorname{ker} \operatorname{ad} X)^{\mathrm{ev}}\right\} .
$$

We recall that $g$ is an algebra of real rank 1 and therefore $g_{Z}$ is generated by elements of the form $v_{4}+v_{0}+v_{-4}$ and $v_{2}+v_{-2}$ where $v_{2}$ and $v_{4}$ centralize ad $X$. If $v_{2}+v_{-2} \in \mathfrak{f}_{Z}$ or $v_{4}+v_{0}+v_{-4} \in \mathfrak{f}_{Z}$ then $\left[H, v_{2}+v_{-2}\right]$ $=2 v_{2}-2 v_{-2}$ and $\left[H, v_{4}+v_{0}+v_{-4}\right]=4 v_{4}-4 v_{-4}$ are in $p$ and have the desired form. The statement follows from these two relations. This also implies that

(2) $\mathfrak{p}_{1}=\left\{Z-\tau Z: Z \in g^{+}\right.$and perpendicular to $\left.(\operatorname{ker} \operatorname{ad} X)^{\mathrm{ev}}\right\}$ where $g^{+}=\sum_{i<0} g_{i}$.

Let $X_{1} \in g^{+}$be such that $W=X_{1}-\tau X_{1} \in \mathfrak{p}_{1}$. Then Theorem 8.11.3 in [14] has as a simple consequence the fact that the group $L$ acts transitively on the unit sphere of the orthogonal of $(\operatorname{ker} \operatorname{ad} X)^{\mathrm{ev}}$. Then in view of the description of $p_{1}$ given in (2) we get $(\mathbf{R} W)^{L}=p_{1}$.

Proposition 4.3. Let $\mathfrak{p}_{Z}^{1}$ be the orthogonal complement of $\mathfrak{p}_{Z}$ in $\mathfrak{p}$. Then the map $\psi: K_{Z} \times \mathbf{R}^{2} \rightarrow \mathfrak{p}_{Z}^{\perp}$ given by $\psi\left(m, s_{1}, s_{2}\right)=\left(s_{1} W+s_{2} H\right)^{m}$ is onto $\mathfrak{p}_{Z}^{\perp}$. 
Proof. We calculate the differential of $\phi$. It equals

$$
D \psi_{\left(m, s_{1}, s_{2}\right)}\left(Z, t_{1}, t_{2}\right)=\operatorname{Ad} m\left(s_{1}[Z, W]+s_{2}[Z, H]+t_{1} W+t_{2} H\right) \text {. }
$$

In view of Corollary 4.2 this map is a submersion at any point $\left(m, s_{1}, s_{2}\right)$ where $s_{1} s_{2} \neq 0$. Let $\mathscr{D}$ be the set of points at which $\psi$ is a submersion. Then (D) is dense in $K_{Z} \times \mathbf{R}^{2}$ and $\psi(\mathcal{D})$ is open in $\mathfrak{p}_{Z}^{\frac{1}{Z}}$. Since $K_{Z}$ is a compact group, $\psi\left(K_{Z} \times \mathbf{R}^{2}\right)$ is also closed. We now examine the boundary of $\psi(\mathscr{D})$. It is contained in $\mathbf{R} W^{K_{z}} \cup \mathbf{R} H^{K_{z}}$ which is a union of two submanifolds namely $\mathbf{R}^{x} W^{K_{z}}$ and $\mathbf{R}^{x} H^{K_{z}}$, and $\{0\}$. If we can show that the two submanifolds have at least codimension 2 in $p_{Z}^{\perp}$ then it follows that $\psi$ is onto. Indeed, let $q \in \mathfrak{p}_{Z}^{\perp} \backslash \psi\left(K_{Z} \times \mathbf{R}^{2}\right)$. Then we can join $q$ to any element of the interior of $\psi\left(K_{Z} \times \mathbf{R}^{2}\right)$ by a curve that does not intersect $\mathbf{R} W^{K_{z}} \cup \mathbf{R} H^{K_{z}}$ (see Proposition 9.4 in [5]). This is a contradiction so $p_{Z}^{\frac{1}{Z}}=\psi\left(K_{Z} \times \mathbf{R}^{2}\right)$.

It remains to show that the boundary has codimension at least 2. By Corollary 4.2 we have the direct sum decomposition

(1) $\mathbf{R} H+\left[H, \mathfrak{f}_{Z}\right]+\mathfrak{p}_{Z}+\mathfrak{p}_{1}=\mathfrak{p}$.

Consider the case where $\operatorname{dim} \mathfrak{p}_{1}>1$ first. Then $\mathbf{R} H+\left[H, \mathfrak{f}_{Z}\right]$ has codimension equal to $\operatorname{dim} \mathfrak{p}_{1}$ so $\left(\mathbf{R}^{x} H\right)^{K_{z}}$ has codimension at least 2 .

We have the following relations.

(2) $B_{\tau}\left(\left[W, \mathfrak{f}_{Z}\right], H\right)=0$,

(3) $B_{\tau}\left(\left[W, \mathfrak{f}_{Z}\right], \mathfrak{p}_{Z}\right)=0$,

(4) $B_{\tau}\left(\left[W, f_{Z}\right], W\right)=0$.

From (2) we obtain that $H$ is orthogonal to $\mathbf{R} W+\left[W, \mathfrak{f}_{z}\right]$. From relation (3) and $W \in \mathfrak{p}_{1}$ we get

(5) $\mathbf{R} W+\left[W, \mathfrak{f}_{z}\right] \subseteq\left[H, \mathfrak{f}_{z}\right]+\mathfrak{p}_{1}$.

In view of $\mathbf{R} W+[W, \mathcal{L}]=\mathfrak{p}_{1}$ let $\left[W, \mathfrak{f}_{Z}\right]^{\perp}$ be the perpendicular complement of $\mathfrak{p}_{1}$ in $\mathbf{R} W+\left[W, \mathfrak{f}_{z}\right]$. Then $\left[W, \mathfrak{f}_{z}\right]^{\perp} \subseteq\left[H, \mathfrak{f}_{z}\right]$. If $\left[W, \mathfrak{f}_{z}\right]^{\perp} \neq\left[H, \mathfrak{f}_{z}\right]$ then we can find an element orthogonal to $\mathbf{R} W+\left[W, \mathfrak{f}_{Z}\right]$ and $H$ so the codimension of $\left(\mathbf{R}^{x} W\right)^{K_{z}}$ is at least 2.

If on the other hand $\left[W, \mathfrak{f}_{Z}\right]^{\perp}=\left[H, \mathfrak{f}_{Z}\right]$ we have equality in (5). Then $\mathbf{R} W+s_{1}\left[W, \mathfrak{t}_{Z}\right]=\left[H, \mathfrak{f}_{Z}\right]+\mathfrak{p}_{1}$ for $s_{1} \neq 0$ so $\left(m, s_{1}, s_{2}\right) \in \mathcal{D}$ for any $s_{1} \neq 0$. Evidently $\{0\}$ has codimension at least 2 .

Next we consider the case $\operatorname{dim} \mathfrak{p}_{1}=1$. Then $W^{L}=W$ and $\mathbf{R} H+\left[H, k_{z}\right]$ is the orthogonal of $\mathbf{R} W+\mathfrak{p}_{Z}$ in $\mathfrak{p}$ so if $s_{2} \neq 0$ then $\left(m, s_{1}, s_{2}\right) \in \mathscr{D}$. Again $\{0\}$ has codimension at least 2 . The proof is now complete.

We now recall the following result.

Proposition 4.4. Let $G$ be a connected semisimple Lie group and let $\mathfrak{g}=\mathfrak{t}+\mathfrak{p}$ be its Cartan decomposition. Let $\mathfrak{p}^{\prime}$ be a linear subspace of $p$ such that $[X,[X, Y]] \in \mathfrak{p}^{\prime}$ for any $X, Y \in \mathfrak{p}^{\prime}$. Let $\mathfrak{p}^{\perp}$ be the orthogonal of $\mathfrak{p}^{\prime}$ in $\mathfrak{p}$. Then $G$ decomposes topologically into $G=K \cdot F \cdot E$ where $F=\exp \mathfrak{p}^{\perp}$ and $E=\exp \mathfrak{p}^{\prime}$. 
Proof. See [9, Theorem 3, p. 40].

By applying this result to the case considered here we get the following corollaries.

Corollary 4.5. Let $F=\exp \mathfrak{p}_{Z}^{\frac{1}{Z}}$ and $S_{Z}=\exp \mathfrak{p}_{Z}$. Then

$$
G=K F S_{Z}=K \exp (\mathbf{R} W+\mathbf{R} H) G_{Z}
$$

Proof. We note that $\mathfrak{p}_{Z}$ is the $\mathfrak{p}$-component of the subalgebra $\mathfrak{g}_{Z}$ so it satisfies the properties of $\mathfrak{p}^{\prime}$ in Proposition 4.4. Thus we can write $G=K F S_{Z}$. By Proposition $4.3 p_{z}^{\perp}=(\mathbf{R} H+\mathbf{R} W)^{K_{z}}$ so

$$
\exp \mathfrak{p}_{Z}^{\frac{1}{Z} \subseteq K_{Z}} \exp (\mathbf{R} H+\mathbf{R} W) K_{Z} .
$$

Therefore, $G=K K_{Z} \exp (\mathbf{R} H+\mathbf{R} W) K_{Z} S_{Z}=K \exp (\mathbf{R} H+\mathbf{R} W) G_{Z}$.

Corollary 4.6. Let $\mathrm{g}=\mathrm{su}(2,1)$,

$$
H=\left[\begin{array}{lll}
0 & 0 & 0 \\
0 & 0 & 1 \\
0 & 1 & 0
\end{array}\right] \text { and } W=\left[\begin{array}{lll}
0 & 0 & 1 \\
0 & 0 & 0 \\
1 & 0 & 0
\end{array}\right] \text {. }
$$

Let $A=\exp \mathbf{R} H$ and $F=\exp \mathbf{R} W$. Then the maps $\phi_{1}, \phi_{2}: A \times F \rightarrow \exp (\mathbf{R} H$ $+\mathbf{R} W)$ given by $\phi_{1}(e, f)=$ fef and $\phi_{2}(e, f)=$ efe are isomorphisms.

PRoof. This corollary can be regarded as a particular case of Proposition 4.4. We give a sketch of the proof. Theorem 1 on p. 37 in [9] shows that $\phi$ maps $A \times F$ into $\exp (\mathbf{R} H+\mathbf{R} W)$. Let $p(t)$ be a path in $\exp (\mathbf{R} H+\mathbf{R} W)$ joining the origin to some arbitrary element $x$. We denote by $|x|$ the Riemannian distance between $x$ and the origin under the metric given by $(d s / d t)^{2}=\operatorname{Tr}\left(p^{-1} \dot{p}\right)$. Then the proof of Theorem 3 in [9] shows that $|e f e|>$ $\sup (|e|,|f|)$. If $e_{n} f_{n} e_{n}$ converges to $x,\left|e_{n} f_{n} e_{n}\right|$ is bounded so $\left|e_{n}\right|$ and $\left|f_{n}\right|$ are also bounded so $e_{n}$ has a convergent subsequence and so does $f_{n}$. Let $e_{n} \rightarrow e \in A$ and $f_{n} \rightarrow f \in F$. Then $e f e=x$. This shows that the image of $\phi$ is closed. A calculation of the Jacobian of $\phi$ shows that the image is also open. The rest of the proof is as in Theorem 1 in [9].

We now put all these results together in the following theorem.

THEOREM 4.7. Let $G$ be a real semisimple group of rank one. With notation as before, $G=K A^{+} \exp \mathbf{R} W G_{Z}$. Let $L_{0}$ be the normalizer of $\mathbf{R} W$ in $L$. Then the map $\psi: K / L_{0} \times \mathbf{R}^{+} \times \mathbf{R}^{\times} \times G_{Z} \rightarrow G$ defined by $\psi\left(k, s_{1}, s_{2}, m\right)=$ $k \exp s_{2} H \exp s_{1} W m$ has finite fiber of constant cardinality.

Proof. Let $x \in G$ be such that $x=k s$ where $k \in K, s \in S$ and $S=$ $\exp p$. Let $x=k_{1} z m$ be the decomposition in Corollary 4.5. By the remark at the end of $\$ 3$ we may assume that the Lie triple $\{X, H, Y\}$ and $W$ are contained in a $\tau$-invariant subalgebra isomorphic to $\operatorname{su}(2,1)$. Let $K^{\prime}$ be the maximal compact subgroup of $\mathrm{SU}(2,1)$ and $G_{Z_{0}}^{\prime}$ be the centralizer of $Z_{0}$ in 
$\mathrm{SU}(2,1)$. In this case, $G_{Z_{0}}^{\prime}$ is compact. We can then write

$$
\begin{aligned}
G & =\left(K / K^{\prime}\right) K^{\prime} \exp (\mathbf{R} H+\mathbf{R} W) G_{Z_{0}}^{\prime}\left(G_{Z_{0}}^{\prime} \backslash G_{Z_{0}}\right) \\
& =\left(K / K^{\prime}\right) \operatorname{SU}(2,1)\left(G_{Z_{0}}^{\prime} \backslash G_{Z_{0}}\right) .
\end{aligned}
$$

In view of this decomposition it is enough to show the result for $\operatorname{SU}(2,1)$. Assume that $x \in \mathrm{SU}(2,1)$. Then $\tau(x)=k s^{-1}$ and $\tau(x)^{-1} x=s^{2}$. Since in this case $G_{Z_{0}}=K_{Z_{0}}, \mathfrak{p}_{Z}^{\perp}=\mathfrak{p}$. Therefore, by Proposition $4.3, s^{2}$ can be conjugated into $\exp (\mathbf{R} H+\mathbf{R} W)$. Let $m \in G_{Z}$ and $Z \in \exp (\mathbf{R} H+\mathbf{R} W)$ be such that $s=m^{-1} z m$. By Corollary 4.6 we can write $z^{2}=f e^{2} f$ with $e \in A$ and $f \in$ $\exp \mathbf{R} W$. Then $x m^{-1} f^{-1} e^{-1} \in K$ since

$$
\begin{aligned}
\tau\left(x m^{-1} f^{-1} e^{-1}\right) & =\tau(x) m^{-1} f e=k s^{-1} m^{-1} f e=k m^{-1} z^{-1} f e \\
& =k m^{-1} z^{-1} z^{2} f^{-1} e^{-1}=k s m^{-1} f^{-1} e^{-1}=x m^{-1} f^{-1} e^{-1} .
\end{aligned}
$$

Let $k^{\prime}=x m^{-1} f^{-1} e^{-1}$. Then $x=k^{\prime}$ efm so $G=K A \exp \mathbf{R} W G_{Z}$.

Suppose that $x=k_{1} e_{1} f_{1} m_{1}=k_{2} e_{2} f_{2} m_{2}$. Then $\tau(x)^{-1} x=m_{1}^{-1} f_{1} e_{1}^{2} f_{1} m_{1}=$ $m_{2}^{-1} f_{2} e_{2}^{2} f_{2} m_{2}$. By Corollary 4.6, $f_{1} e_{1}^{2} f_{1}$ and $f_{2} e_{2}^{2} f_{2}$ are elements of $\exp (\mathbf{R} H+$ $\mathbf{R} W)$ so we have to investigate the relationship $\left(t_{1} W+t_{2} H\right)^{m}=s_{1} W+s_{2} H$ where $t_{1}$ and $t_{2}$ are nonzero. Due to the proof of Proposition 4.3, $\left(t_{1} W+\right.$ $\left.t_{2} H\right)^{K_{z}}$ is transverse to $\mathbf{R} H+\mathbf{R} W\left(t_{1}\right.$ and $t_{2}$ are fixed) since $\left[W, \mathfrak{f}_{Z}\right]$ and $\left[H, \mathfrak{f}_{z}\right]$ are both orthogonal to $\mathbf{R} H+\mathbf{R} W$.

This also implies that the intersection has dimension 0 . Since $K_{Z}$ is compact, $\left(t_{1} W+t_{2} H\right)^{K_{z}} \cap(\mathbf{R} H+\mathbf{R} W)$ is a finite set. We have to show that it has constant cardinality and also compute the dimension of the centralizer of $t_{1} W+t_{2} H$ in $K_{Z}$. Let $\mathcal{L}_{0}$ be the Lie algebra of $L_{0}$. There are two cases.

Case I. ad $H$ has odd eigenvalues. In this case $K_{Z}$ leaves $\mathfrak{p}_{1}$ invariant since $\mathfrak{f}_{Z} \subseteq g^{\mathrm{ev}}$ while $\mathfrak{p}_{1}$ is generated by elements of the form $X_{1}-\tau X_{1}$ where $X_{1} \in g^{\mathrm{ev}}$. Let $m \in K_{Z}$. If $\left(t_{1} W+t_{2} H\right)^{m}=s_{1} W+s_{2} H$ then $t_{1} W^{m}=s_{1} W$ and $t_{2} H^{m}=s_{2} H$. This implies that the connected component of the centralizer of $t_{1} W+t_{2} H$ is contained in $L_{0}$. Since $L$ acts transitively on the unit sphere in $g$ we can find $l \in L$ such that $W^{l}=-W$. Then $\left(t_{1} W+t_{2} H\right)^{l}=$ $\left(-t_{1} W+t_{2} H\right)$. Also

$$
\begin{aligned}
\operatorname{card}\left\{\left(t_{1} W\right.\right. & \left.\left.+t_{2} H\right)^{K_{z}} \cap(\mathbf{R} W+\mathbf{R} H)\right\} \\
& =\operatorname{card}\left\{\left(-t_{1} W-t_{2} H\right)^{K_{z}} \cap(\mathbf{R} W+\mathbf{R} H)\right\} .
\end{aligned}
$$

Since the map $\phi: K_{Z} \times\left(\mathbf{R}^{x}\right)^{2} \rightarrow \mathfrak{p}_{Z}^{\frac{1}{Z}}$ is a submersion, $\phi$ has constant fibers on each connected component of $\left(\mathbf{R}^{x}\right)^{2}$.

Case II. ad $H$ has even eigenvalues only. We calculate the dimension of $K / L_{0} \times A \times \exp \mathbf{R} W \times G_{Z}$. We already know that the map $\phi$ is a submersion. We then have the following relations

(1) $\operatorname{dim} \mathfrak{f}=\operatorname{dim} g_{0}-1+\operatorname{dim} g_{2}+\operatorname{dim} g_{4}$,

(2) $\operatorname{dim} g_{Z}=\operatorname{dim} g_{0}$ 
(3) $\operatorname{dim} \mathfrak{L}-\operatorname{dim} \varrho_{0}=\operatorname{dim} \mathfrak{p}_{1}-1$,

(4) $\operatorname{dim} \mathfrak{p}_{1}+\operatorname{dim} \mathfrak{p}_{Z}+\operatorname{dim}\left[H, \mathfrak{f}_{Z}\right]+1=\operatorname{dim} \mathfrak{p}=\operatorname{dim} g_{2}+\operatorname{dim} g_{4}+1$,

(5) $\operatorname{dim}\left[H, \mathfrak{f}_{Z}\right]=\operatorname{dim} \mathfrak{f}_{Z}-\operatorname{dim} \mathcal{L}$.

Then relations (3), (4) and (5) give

$$
\operatorname{dim} \mathfrak{L}_{0}=\operatorname{dim} \mathcal{L}-\operatorname{dim} \mathfrak{p}_{1}+1=\operatorname{dim} g_{0}-\operatorname{dim} g_{2}-\operatorname{dim} g_{4}+1
$$

so

$$
\operatorname{dim} f-\operatorname{dim} \mathfrak{l}_{0}+2+\operatorname{dim} g_{Z}=\operatorname{dim} g_{0}+2 \operatorname{dim} g_{2}+2 \operatorname{dim} g_{4}=n .
$$

To show that the fiber has constant cardinality we make use of the fact that the map $\phi$ mentioned in Case $I$ is a submersion so its fiber has constant cardinality on connected components of $\left(\mathbf{R}^{x}\right)^{2}$, and the relation

$$
\operatorname{card}\left(\left(-t_{1} W-t_{2} H\right)^{L_{0}}\right)=\operatorname{card}\left(\left(t_{1} W+t_{2} H\right)^{L_{0}}\right) .
$$

Let $X_{2}=\left[X_{1}, X\right] \in g_{4}$ where $X_{1}-\tau X_{1}=W$. Then $X_{2} \neq 0$ and there is $l$ centralizing $X_{2}$ and $H$ (by Theorem 8.11.3 in [14]) such that $X^{l}=-X$. But then $W^{l}=-W$. Let $\left(t_{1} W+t_{2} H\right)^{m}=s_{1} W+s_{2} H$ where $m \in L_{0}$. Then $l^{-1} m l \in L_{0}$ and

$$
\left(-t_{1} W+t_{2} H\right)^{l^{-1} m l}=\left(t_{1} W+t_{2} H\right)^{m l}=\left(s_{1} W+s_{2} H\right)^{l}=-s_{1} W+s_{2} H .
$$

Thus the fiber has constant cardinality. The proof of the proposition is complete once we note that in $\operatorname{su}(2,1)$ the element

$$
\left[\begin{array}{rrr}
-1 & 0 & 0 \\
0 & -1 & 0 \\
0 & 0 & +1
\end{array}\right] \in L_{0}
$$

maps $H$ into $-H$.

We now derive an integration formula corresponding to the decomposition in Theorem 4.7.

Proposition 4.8. Let $\psi: K / L_{0} \times A^{+} \times \exp \mathbf{R} W \times G_{Z} \rightarrow G$ be the map given by $\psi(k, a, w, m)=$ kawm. Let $g\left(t_{1}, t_{2}\right)=\left|\operatorname{det} D \psi_{k, a, w, m}\right|$ where $k \in$ $K / L_{0}, a=\exp t_{2} H, w=\exp t_{1} W$ and $m \in G_{Z}$. Let $c_{Z_{0}}$ be the cardinality of the fiber of $\psi$ at any point where $t_{1}, t_{2} \neq 0$. Then

$$
\int_{G / G_{Z_{0}}} f\left(Z_{0}^{x}\right) d x=\frac{1}{c_{Z_{0}}} \int_{K / L_{0}} \int_{A^{+}} \int_{\mathbf{R}} g\left(t_{1}, t_{2}\right) f\left(Z_{0}^{k a w}\right) d t_{1} d a d k .
$$

Furthermore $g\left(t_{1}, t_{2}\right)=|\operatorname{det} \phi|$ where $\phi$ is the linear map

$$
\phi\left(Z_{1}, s_{2}, s_{1}, Z_{2}\right)=\operatorname{Ad} a^{-1} Z_{1}+s_{2} H+s_{1} W+\operatorname{Ad} w^{-1} Z_{2}
$$

for $Z_{1} \in \mathfrak{f} / \complement_{0}, Z_{2} \in g_{Z}$.

Proof. In order to establish the integral formula it is enough to calculate the pullback under $\psi$ of the Haar measure $d x$ of $G$. 
By Theorem 4.7, $K / L_{0} \times A^{+} \times \exp \mathbf{R}^{\times} W \times G_{Z}$ is a covering space of an open dense subspace of $G$ and the fiber has constant cardinality. Then the following formula holds for any $f \in C_{c}^{\infty}(G)$.

$$
\int_{G} f(x) d x=\frac{1}{c_{Z_{0}}} \int_{K / L_{0}} \int_{A^{+}} \int_{\mathbf{R}} \int_{G_{Z}} f(k a w m) J_{k, a, w, m} d k d a d w d m
$$

where $J$ is the Jacobian. Due to the fact that the measures involved are all left and right invariant we can express $J_{k, a, w, m}$ as a function of $a$ and $w$ alone. We get

$$
\begin{aligned}
D \psi_{k, a, w, m}\left(Z_{1}, s_{2}, s_{1}, Z_{2}\right)=L_{k a w m}^{*} & \left(\operatorname{Ad}\left(m^{-1} w^{-1} a^{-1}\right) Z_{1}\right. \\
& \left.+s_{2} \operatorname{Ad}\left(m^{-1} w^{-1}\right) H+s_{1} \operatorname{Ad}\left(m^{-1}\right) W+Z_{2}\right)
\end{aligned}
$$

where $L_{g}^{*}$ is the differential of left multiplication by $g$. By using the fact that $d x$ is left invariant and that $|\operatorname{det} \operatorname{Ad} x|=1$ for any $x \in G$ and $\left|\operatorname{det}_{g_{z}} \operatorname{Ad}(m)\right|$ $=1$ for any $\mathfrak{m} \in G_{Z}$ we get $\left|J_{k, a, w, m}\right|=\mid \operatorname{det}\left(\operatorname{Ad} a^{-1} Z_{1}+s_{2} H+s_{1} W+\right.$ Ad $\left.w Z_{2}\right) \mid$. This is exactly the expression for $|\operatorname{det} \phi|$ in the statement of the proposition. The integral formula now follows from the normalization of the measures so that $d x=d_{G / G_{z}} x^{*} \cdot d_{G_{z}} \dot{x}$.

5. The orbit of the nilpotent element $X$. We now find a decomposition and an integral formula similar to Theorem 4.7 and Proposition 4.8 for a nilpotent element $X$. Let $\{X, H, Y\}$ be a Lie triple as before.

Lemma 5.1. Let $N_{0}=\operatorname{Cent}_{N} X$. If $G_{X}$ is the centralizer of $X$ in $G$ then $G_{X}=L N_{0}$. Furthermore, if $y \in G_{X}$ is semisimple then $y$ can be conjugated into $L$ by some element in $N_{0}$.

Proof. From representation theory of $\operatorname{sl}(2, \mathrm{C})$ it follows that Cent $\mathrm{g}_{\mathrm{g}} X=\mathfrak{L}$ $+n_{0}$ where $n_{0}$ is the Lie algebra of $N_{0}$. Let $y \in G$ be such that $X^{y}=X$. Then $H^{y}-H \in \mathrm{Cent}_{\mathrm{g}} X$. Therefore, $H^{y}=H+m_{0}+n_{0}$ where $m_{0} \in \mathcal{L}$ is semisimple and $n \in \mathfrak{n}_{0}$. For any invariant polynomial $p$ we have the relation $p(H)=p\left(H^{y}\right)=p\left(H+m_{0}+n_{0}\right)=p\left(H+m_{0}\right)$. But ad $m_{0}$ has imaginary eigenvalues and $\left[H, m_{0}\right]=0$. It follows that $m_{0}=0$. By a well-known argument, $H+n_{0}=H^{n_{1}}$ for some $n_{1} \in N_{0}$. Thus $H^{y}=H^{n_{1}}$ so $y n_{1}^{-1} \in \operatorname{Cent}_{G} H$ $\cap$ Cent $_{G} X=L$. This establishes the decomposition $G_{X}=L N_{0}$.

Let now $y \in G_{X}$ be semisimple. Ad $y$ normalizes $N_{0}$ so

$$
\mathrm{n}_{0}=\operatorname{Im}(-\operatorname{Ad} y+1) \oplus \operatorname{ker}(-\operatorname{Ad} y+1) .
$$

Then $H^{y}=H+Z_{0}+Z_{1}$ where $Z_{0}=(-\operatorname{Ad} y+1) Z_{2}$ and $(-\operatorname{Ad} y+1) Z_{1}$ $=0$. Then

$$
\left(H+Z_{2}\right)^{y}=H+Z_{0}+Z_{1}+Z_{2}^{y}=H+Z_{2}+Z_{1}
$$


so that

$$
(\operatorname{Ad} y-1)^{2}\left(H+Z_{2}\right)=(\operatorname{Ad} y-1) Z_{1}=0 \text {. }
$$

Since Ad $y-1$ is semisimple $($ Ad $y-1)\left(H+Z_{2}\right)=0$. As $H+Z_{2}=H^{n}$ for some $n \in N_{0}$ it follows that $H^{n}=H+Z_{2}=\left(H+Z_{2}\right)^{y}=H^{n y}$ or $n y n^{-1} \in$ Cent $_{G} H \cap$ Cent $_{G} X=L$.

This completes the proof.

LEMMA 5.2. Let $\mathrm{n}_{0}$ be the Lie algebra of $N_{0}$ and $n_{1}$ its orthogonal complement in n. If $N_{1}=\exp \mathfrak{n}_{1}$ then $N=N_{1} N_{0}$ and $G=K / L A N_{1} G_{X}$.

Proof. Let $\mathfrak{n}^{(k)}=\sum_{i \geqslant k} g_{i}$. Then $\mathfrak{n}^{(k)}$ is an ideal in $\mathfrak{n}, \mathfrak{n}^{(k)} \supseteq \mathfrak{n}^{(k+1)}$ and $\mathfrak{n}^{(1)}=n$. Furthermore, since ad $H \mathfrak{n}_{0} \subseteq \mathfrak{n}_{0}$ and ad $H \mathfrak{n}_{1} \subseteq \mathfrak{n}_{1}$ we can decompose $\mathfrak{n}^{(k)}$ into $\mathfrak{n}^{(k)}=\mathfrak{n}^{(k)} \cap \mathfrak{n}_{0}+\mathfrak{n}^{(k)} \cap \mathfrak{n}_{1}$. Thus Lemma 1 on p. 736 in [4a] applies so $N=N_{1} N_{0}$. To show that this decomposition is unique it is enough to show that, if $\exp X_{2}=\exp \left(-X_{1}\right) \exp Y_{1}$ where $X_{2} \in \mathfrak{n}_{0}$ and $X_{1}, Y_{1} \in \mathfrak{n}_{1}$, then $X_{2}=0$. The Campbell-Baker-Hausdorff formula states that

$$
\begin{aligned}
\log \left[\exp \left(-X_{1}\right) \exp Y_{1}\right]= & -X_{1}+Y_{1}+\frac{1}{2}\left[-X_{1}, Y_{1}\right] \\
& +\frac{1}{12}\left[-X_{1},\left[-X_{1}, Y_{1}\right]\right]+\cdots .
\end{aligned}
$$

Suppose that $X_{1}=Z+X_{1}^{\prime}$ where $X_{1}^{\prime}=\mathfrak{n}^{(s+1)}$ and $Y_{1}=Z+Y_{1}^{\prime}$ where $Y_{1}^{\prime} \in$ $\mathfrak{n}^{(s+1)}$. Then $\left[X_{1}, Y_{1}\right]=\left[Z, Y_{1}^{\prime}\right]+\left[X_{1}^{\prime}, Z\right]+\left[X_{1}^{\prime}, Y_{1}^{\prime}\right] \in \mathfrak{n}^{(s+2)}$. Therefore

$$
\log \left[\exp \left(-X_{1}\right) \exp Y_{1}\right] \equiv Y_{1}^{\prime}-X_{1}^{\prime}\left(\bmod \mathfrak{n}^{(2+s)}\right)
$$

On the other hand $\log \left[\exp \left(-X_{1}\right) \exp Y_{1}\right] \equiv X_{2}\left(\bmod \mathfrak{n}^{(s+2)}\right)$ which is possible only if $X_{1}^{\prime} \equiv Y_{1}^{\prime} \bmod \mathfrak{n}^{(s+2)}$ and $X_{2} \equiv 0 \bmod \mathfrak{n}^{(s+2)}$. A simple induction argument then shows that $X_{2}=0$. The Iwasawa decomposition transforms into

$$
G=K A N=K A N_{1} N_{0}=K / L L A N_{1} N_{0}=K / L A N_{1} L N_{0}=K / L A N_{1} G_{X}
$$

and the proof is complete.

REMARK. The previous two lemmas are true for $G$ of arbitrary rank with minor modifications.

In accordance with $\$ 4$ let $W=X_{1}-\tau X_{1}$ where $X_{1} \in \mathfrak{n}$.

Proposition 5.3. Let $G$ be a semisimple Lie group of real rank one. Then $G=K A \exp \mathbf{R} X_{1} G_{X}$ and the decomposition is unique up to $L_{0}=\operatorname{Norm}_{L}\left\{\mathbf{R} X_{1}\right\}$ $=\operatorname{Norm}_{L}\{\mathbf{R} W\}$.

Proof. By the proof of Corollary 4.2 we know that $\mathrm{n}_{1}=\left(\mathbf{R} X_{1}\right)^{L}$ so $N_{1}=\left(\exp \mathbf{R} W_{1}\right)^{L}$. We substitute this into the decomposition of Lemma 5.2.

$$
G=K / L A\left(\exp \mathbf{R} X_{1}\right)^{L} G_{X}=K A \exp \mathbf{R} X_{1} G_{X} .
$$


Suppose $k_{1} a_{1} \exp t_{1} X_{1} l_{1} n_{1}=k_{2} a_{2} \exp t_{2} X_{1} l_{2} n_{2}$. Then

$$
k_{1} l_{1} a_{1} \exp t_{1} X_{1}^{l^{-1}} n_{1}=k_{2} l_{2} a_{2} \exp t_{2} X_{1}^{l^{-1}} n_{2} \text {. }
$$

Since this decomposition is unique, $a_{1}=a_{2}, k_{1} l_{1}=k_{2} l_{2}$, exp $t_{1} X_{1}^{l^{-1}}=$ $\exp t_{2} X_{1}^{l^{-1}}$ or $\left(t_{1} X_{1}\right)^{l_{1}^{-1} l_{2}}=t_{2} X_{1}$ and $n_{1}=n_{2}$. Thus $l_{1} l_{2}^{-1} \in \operatorname{Norm}_{L}\left(\mathbf{R} W_{1}\right)$ so $k_{2}^{-1} k_{1} \in \operatorname{Norm}_{L}\left(\mathbf{R} X_{1}\right)$. Thus the decomposition $G=K / L_{0} A \exp t_{1} X_{1} G_{X}$ is unique.

Proposition 5.4. Let $\rho(H)=\left.\frac{1}{2} \operatorname{tr} \operatorname{ad} H\right|_{\mathfrak{n}}$. Then

$$
\int_{G / G_{X}} f\left(X^{x}\right) d x=\int_{K / L_{0}} \int_{A} e^{2 \rho(\log a)} \int_{\mathbf{R}} t^{\operatorname{dim} n_{1}-1} f\left(X^{k a \exp t_{1} X_{1}}\right) d t_{1} d a d k
$$

for any $f \in C_{c}^{\infty}(g)$.

Proof. Let $\psi: K / L_{0} \times A \times \exp t_{1} W \times G_{X} \rightarrow G$ be the map given by $\psi(k, a, w, m)=k a w m$ where $w=\exp t_{1} X_{1}$ and $m \in G_{X}$.

As in Proposition 4.8 we have to calculate the pullback under $\psi$ of the Haar measure $d x$ of $G$. The map $\psi$ is an isomorphism so for any $f \in C_{c}^{\infty}(G)$,

$$
\int_{G} f(x) d x=\int_{K / L_{0}} \int_{A} \int_{\mathbf{R}} \int_{G_{X}} f(k a w m) J_{k, a, w, m} d k d a d t_{1} d m
$$

where $J$ is the Jacobian. We obtain the formula

$$
\begin{aligned}
D \psi_{k, a, w, m}\left(Z_{1}, s_{2}, s_{1}, Z_{2}\right)=L_{k a w m}^{*} & \left(\operatorname{Ad}\left(m^{-1} w^{-1} a^{-1}\right) Z_{1}\right. \\
& \left.+s_{2} \operatorname{Ad}\left(m^{-1} w^{-1}\right) H+s_{1} \operatorname{Ad}\left(m^{-1}\right) X_{1}+Z_{2}\right) .
\end{aligned}
$$

By using the fact that $G_{X}$ is unimodular the same argument as in Proposition 4.8 implies

$$
J_{k, a, w, m}=\left|\operatorname{det}\left(\operatorname{Ad} a^{-1} Z_{1}+s_{2} H+s_{1} X_{1}+\operatorname{Ad} w Z_{2}\right)\right| .
$$

We observe that

$$
\text { Ad } w=e^{t_{1} \text { ad } X_{1}}=I+t_{1} \text { ad } X_{1}+t_{1}^{2}\left(\operatorname{ad} X_{1}\right)^{2} / 2+\cdots
$$

so in view of the fact that $X_{1}$ is nilpotent, we have

(1) $\left.\mathrm{Ad} w\right|_{\mathfrak{L}_{0}}=\mathrm{id}$

(2) Ad $w Z_{2}=Z_{2}+t_{1}\left[Z_{2}, X_{1}\right]+$ higher order terms $\left(Z_{2} \in \mathcal{L} / \mathscr{L}_{0}\right)$,

(3) Ad $w Z_{2}=Z_{2}+$ higher order terms if $Z_{2} \in \mathfrak{n}_{0}$.

Then Ad $w$ maps $\mathcal{L}+n_{0}$ into $\mathcal{L}+n$ and is given by upper triangular matrices.

On the other hand has a basis formed by vectors $Z+\tau Z$ where $Z \in \mathfrak{n}$ and vectors $Z^{\prime} \in \mathrm{Cent}_{\mathrm{f}} H$. Also the map $Z_{2} \rightarrow\left[Z_{2}, X_{1}\right]$ is onto the orthogonal complement of $X_{1}$ in $\mathfrak{n}_{1}$. By putting all these facts together we can calculate the determinant of $D \psi$. It equals $t_{1}^{\operatorname{dim} n_{1}-1} e^{2 \rho(\log a)}$ where the power of $t_{1}$ comes from relation (2) and $e^{2 \rho(\log a)}$ comes from the action $\operatorname{Ad} a^{-1}$ on $f$. 
Finally the formula in the proposition is a consequence of the normalization of the measures so that $d x=d_{G / G_{X}} \dot{x} d_{X} x^{*}$.

6. The relationship between the unipotent and semisimple integrals. We use the same notation as before. Let $G_{Z_{0}}=\operatorname{Cent}_{G} Z_{0}$ and $G_{X}=\operatorname{Cent}_{G} X$. The goal of this section is to prove the formula in Theorem 6.5.

By Remark 3.3 we may assume that $X, H, Y, W \in \mathrm{su}(2,1) \subseteq g$. According to the nature of the eigenvalues of ad $H$ there are two cases.

Case I. ad $H$ has even eigenvalues only. This is a special case of some results on even nilpotent elements of $R$. Rao. We give an independent argument. We may set

$$
\begin{aligned}
& X=\left[\begin{array}{rrr}
0 & -i & -i \\
-i & 0 & 0 \\
+i & 0 & 0
\end{array}\right], \quad Y=\left[\begin{array}{rrr}
0 & -i & i \\
-i & 0 & 0 \\
-i & 0 & 0
\end{array}\right], \\
& H=\left[\begin{array}{rrr}
0 & 0 & 0 \\
0 & 0 & -2 \\
0 & -2 & 0
\end{array}\right] \text { and } W=\left[\begin{array}{lll}
0 & 0 & 1 \\
0 & 0 & 0 \\
1 & 0 & 0
\end{array}\right]
\end{aligned}
$$

We obtain the following relation

$$
Z_{0}^{\exp t_{1} W}=i\left[\begin{array}{ccc}
2 \sinh 2 t_{1} & 0 & -2 \cosh 2 t_{1} \\
0 & 0 & 0 \\
2 \cosh 2 t_{1} & 0 & -2 \sinh 2 t_{1}
\end{array}\right] .
$$

Then

$$
Z_{0}^{\exp t_{1} W}=\cosh 2 t_{1} Z_{0}+\sinh 2 t_{1} X_{0}+\sinh 2 t_{1}\left(X_{2}-\tau X_{2}\right),
$$

where

$$
X_{0}=\left[\begin{array}{rrr}
2 i & 0 & 0 \\
0 & -i & 0 \\
0 & 0 & -i
\end{array}\right]
$$

is in $g_{0}$ and

$$
X_{2}=\left[\begin{array}{rrr}
0 & 0 & 0 \\
0 & -i / 2 & i / 2 \\
0 & -i / 2 & i / 2
\end{array}\right]
$$

is in $g_{4}$. Then

$$
\begin{aligned}
t Z_{0}^{\exp t_{1} W}=\left[\cosh 2 t_{1}\left(X-t^{2} Y\right)+t \sinh 2 t_{1} X_{0}\right. & \\
& \left.+\sinh 2 t_{1}\left(\frac{1}{t} X_{2}-t^{3} \tau X_{2}\right)\right]^{\exp \theta H}
\end{aligned}
$$

where $\theta=\frac{1}{2} \log t$. 
In the notation of Proposition 4.8 we consider

$$
\int_{0}^{\infty} \int_{0}^{\infty} g\left(t_{1}, t_{2}\right) f\left(t Z_{0}^{a w}\right) d t_{1} d t_{2}
$$

and by using relations (1) and (2) we make the change of variables $t_{2}^{1}=t_{2}+$ $\theta$ and $t_{1}^{1}=t_{1} / t$. The integral transforms into

$$
\int_{0}^{\infty} \int_{(1 / 2) \log t}^{\infty} \operatorname{tg}\left(t t_{1}, t_{2}-\theta\right) f\left(t, t_{1}, t_{2}\right) d t_{1} d t_{2}
$$

where

$$
f\left(t, t_{1}, t_{2}\right)=f\left(\frac{1}{t} \sinh 2 t t_{1} X_{2}+Z_{t, t_{1}}\right)^{\exp t_{2} H} .
$$

The term $Z_{t, t_{1}}$ is equal to

$$
Z_{t, t_{1}}=\cosh 2 t t_{1} X+t \sinh 2 t t_{1} X_{0}-t^{2} \cosh 2 t t_{1} Y-t^{3} \sinh 2 t t_{1} \tau X_{2} .
$$

LEMMA 6.1. The function $f\left(t, t_{1}, t_{2}\right)$ has compact support in $t_{1}$ and $t_{2} \geqslant 0$ independent of $t$.

Proof. From formula (2) we see that $Z_{t, t_{1}}$ is orthogonal to $X_{2}$ with respect to $-B_{\tau}$. Then

$$
\left\|\left(\frac{1}{t} \sinh 2 t t_{1} X_{2}+Z_{t, t_{1}}\right)^{\exp t_{2} H}\right\|^{2} \geqslant e^{8 t_{2}} \frac{1}{t^{2}}\left(\sinh 2 t t_{1}\right)^{2}\left\|X_{2}\right\|^{2} .
$$

Since $\left(\left(t t_{1}\right)^{-1} \sinh 2 t t_{1}\right)^{2} \geqslant C>0$ for any $t$ and $t_{1}$ we can write

$$
\left\|\left(\frac{1}{t} \sinh 2 t t_{1} X_{2}+Z_{t, t_{1}}\right)^{\exp t_{2} H}\right\|^{2}>C t_{1}^{2} e^{8 t_{2}}
$$

for any $t$. Since $f$ has compact support, the proof is complete.

We rewrite the integral in (3) as

$$
\begin{aligned}
t \int_{0}^{\infty} \int_{(1 / 2) \log t}^{0} g\left(t t_{1}, t_{2}-\theta\right) f\left(t, t_{1}, t_{2}\right) d t_{1} d t_{2} \\
\quad+t \int_{0}^{\infty} \int_{0}^{\infty} g\left(t t_{1}, t_{2}-\theta\right) f\left(t, t_{1}, t_{2}\right) d t_{1} d t_{2} .
\end{aligned}
$$

Lemma 6.2. Let $r=\operatorname{dim}$ Cent $X$ and $n-\operatorname{dim} g$. Then

$$
2 \rho(H)=n-r+2\left(\operatorname{dim} \mathcal{L}-\operatorname{dim} \varrho_{0}+1\right)=n-r+2 \operatorname{dim} \mathfrak{p}_{1} .
$$

Proof. We have $2 \rho(H)=2 \operatorname{dim} g_{2}+4 \operatorname{dim} g_{4}$. Since the map ad $W$ : $\mathcal{L} \rightarrow \mathfrak{p}_{1}$ is onto the orthogonal complement of $W$ we get

$$
\operatorname{dim} \mathfrak{L}-\operatorname{dim} \mathfrak{L}_{0}+1=\operatorname{dim} \mathfrak{p}_{1} \text {. }
$$


Also

$$
\begin{gathered}
n=\operatorname{dim} g_{0}+2\left(\operatorname{dim} g_{2}+\operatorname{dim} g_{4}\right), \\
r=\operatorname{dim} \mathcal{L}+\operatorname{dim} g_{2}-\operatorname{dim} \mathfrak{p}_{1}+\operatorname{dim} g_{4}=\operatorname{dim} g_{0} \quad \text { and } \\
\operatorname{dim} g_{4}=\operatorname{dim} \mathfrak{p}_{1}
\end{gathered}
$$

since ad $X: g_{2} \rightarrow g_{4}$ is onto and ad $H$ is even. Thus

$$
\begin{aligned}
n-r+2 \operatorname{dim} \mathfrak{p}_{1} & =\operatorname{dim} g_{0}+2\left(\operatorname{dim} g_{2}+\operatorname{dim} g_{4}\right)-\operatorname{dim} g_{0}+2 \operatorname{dim} \mathfrak{p}_{1} \\
& =2 \operatorname{dim} g_{2}+4 \operatorname{dim} g_{4}
\end{aligned}
$$

and the proof of the lemma is complete.

Proposition 6.3. For any $f \in C_{c}(g)$,

$$
\lim _{t \rightarrow 0^{+}} t^{(n-r) / 2} \int_{G / G_{Z_{0}}} f\left(t Z_{0}^{x}\right) d x=c_{X} \int_{G / G_{X}} f\left(X^{x}\right) d x
$$

where $c_{X}$ depends only on the conjugacy class of $X$.

Proof. We note that in formula (3) $g \neq 0$ so we may replace $g$ by det $\phi$ where $\phi$ has the expression given in Proposition 4.8. The same holds for formula (8). We note that

$$
\lim _{t \rightarrow 0}\left(\frac{1}{t} \sinh 2 t t_{1} X_{2}+\cosh 2 t t_{1} X+Z_{t, t_{1}}^{\prime}\right)=X+2 t_{1} X_{2}=X^{\exp t_{1} X_{1}}
$$

where $X_{1}$ is defined by $X_{1}-\tau X_{1}=W, X_{1} \in g_{2}$ and

$$
Z_{t, t_{1}}^{\prime}=Z_{t, t_{1}}-\cosh 2 t t_{1} X \text {. }
$$

We then have to calculate $\lim _{t \rightarrow 0^{+}} t^{(n-r) / 2+1} g\left(t t_{1}, t_{2}-\theta\right)$. We choose a basis of $\mathfrak{t} / \mathscr{L}_{0}$ consisting of $X_{i}+\tau X_{i}$ and $Y_{j}$, where $X_{i} \in g_{i}$ with $i>0$ and $Y_{j} \in \mathcal{E}$ orthogonal to $\mathfrak{L}_{0}$. For $\mathfrak{g}_{Z}$ we choose an orthogonal basis consisting of $Y_{j}$ and $Z_{k}$ where $Y_{j}$ are as before. Then

$$
\begin{array}{rl}
t^{(n-r) / 2+1} & g\left(t t_{1}, t_{2}-\theta\right) \\
= & t^{(n-r) / 2+1} \operatorname{det}\left(e^{-i t_{2} i / 2} X_{i}+e^{i t_{2}-i / 2} \tau X_{i}, Y_{j}, H, W, \operatorname{Ad} w Y_{j}, \operatorname{Ad} w Z_{k}\right) \\
= & t_{1}^{\operatorname{dim~} p_{1}-1} \operatorname{det}\left(e^{-i t_{2}{ }^{i} X_{i}}+e^{i t_{2}} X_{i}, Y_{j}, H, W, \frac{\operatorname{Ad} w Y_{j}-Y_{j}}{t t_{1}}, \operatorname{Ad} w Z_{k}\right)
\end{array}
$$

by using the formula for $n-r$ in Lemma 6.2. Since $w=\exp t t_{1} W$,

$$
\lim _{t \rightarrow 0^{+}} \frac{\operatorname{Ad} w Y_{j}-Y_{j}}{t t_{1}}=\left[W, Y_{j}\right]
$$

Thus

$$
\begin{aligned}
& \lim _{t \rightarrow 0} t^{(n-r) / 2+1} g\left(t t_{1}, t_{2}-\theta\right) \\
& \quad=t_{1}^{\operatorname{dim} p_{1}-1} e^{2 t_{2} \rho(H)} \operatorname{det}\left(\tau X_{i}, Y_{j}, H, W,\left[W, Y_{j}\right], Z_{k}\right) \\
& \quad=\operatorname{const} t_{1}^{\operatorname{dim} p_{1}-1} e^{2 t_{2} \rho(H)} .
\end{aligned}
$$


Since $f\left(t, t_{1}, t_{2}\right)$ has compact support in $t_{1}$ and $t_{2}>0$ independent of $t$, the second integral in (8) converges to

$$
\int_{0}^{\infty} \int_{0}^{\infty} e^{2 \rho(\log a)} t_{1}^{\operatorname{dim} p_{1}-1} f\left(X^{\exp t_{1} X_{1}}\right) d t_{1} d t_{2}
$$

by the dominated convergence theorem. For the term for which $t_{2}<0$, we expand the determinant into powers of $e^{t_{2}}$. For positive powers of $e^{t_{2}}$ we get terms of the form $e^{j t_{2} t(2 \rho(H)-j) / 2}$ where $0<j \leqslant 2 \rho(H)$. All these terms are absolutely integrable on $(-\infty, 0)$ so we may take the limit inside the integral sign. All terms that have $j<2 \rho(H)$ converge to 0 while for $j=2 \rho(H)$ we get

$$
e^{2 t_{2} \rho(H)} t^{\operatorname{dim} p_{1}-1} \operatorname{det}\left(\tau X_{i}, Y_{j}, H, W,\left[W, Y_{j}\right], Z_{k}\right) .
$$

For strictly negative powers of $e^{t_{2}}$ we make a change of variables $u=t_{2}-$ $\log t$. The integral becomes a sum of terms

$$
\begin{aligned}
& \int_{0}^{\infty} \int_{-(1 / 2) \log t}^{\infty} t_{1}^{\operatorname{dim} p_{1}-1} e^{j u} t^{(2 \rho(H)+j) / 2} \\
& \cdot \operatorname{det}\left(\tilde{X}_{i}, Y_{j}, H, W, \frac{\operatorname{Ad} w Y_{j}-Y_{j}}{t t_{1}}, \operatorname{Ad} w Z_{k}\right) \tilde{f}\left(t, t_{1}, t_{2}\right) d t_{1} d t_{2}
\end{aligned}
$$

where $\tilde{X}_{i}$ are either $X_{i}$ or $\tau X_{i}$. Since $j<0, e^{j u}$ is absolutely integrable and $\tilde{f}\left(t, t_{1}, t_{2}\right)$ is bounded in the interval $(0, \infty)$, all the terms converge to 0 (we note that $-\frac{1}{2} \log t \rightarrow+\infty$ ).

Finally the term for $j=0$ can be estimated by

$$
t^{\rho(H)} \int_{0}^{\infty} \int_{-(1 / 2) \log t}^{0} f\left(t, t_{1}, t_{2}\right) d t_{1} \leqslant C t^{\rho(H)}|\log t| .
$$

Since $\rho(H) \geqslant 1$ this term also converges to 0 .

Putting all these facts together we get the formula

$$
\begin{aligned}
\lim _{t \rightarrow 0^{+}} t^{(n-r) / 2} \int_{0}^{\infty} \int_{0}^{\infty} g\left(t_{1}, t_{2}\right) f\left(t Z_{0}^{a w}\right) d t_{1} d t_{2} \\
\quad=\int_{0}^{\infty} \int_{-\infty}^{\infty} e^{2 \rho(\log a)} t_{1}^{\operatorname{dim} p_{1}-1} f\left(X^{\exp t_{1} X_{1}}\right) d t_{1} d t_{2} .
\end{aligned}
$$

In Proposition 4.8 we can split the right-hand side of the formula into a sum of two integrals each of which converges to the corresponding expression to the right-hand side of (9). We thus get 


$$
\begin{aligned}
\lim _{t \rightarrow 0^{+}} t^{(n-r) / 2} \int_{G / G_{Z_{0}}} f\left(t Z_{0}^{x}\right) d x & \\
= & \frac{1}{c_{Z_{0}}} \int_{K / L_{0}} \int_{A} \int_{\mathbf{R}} e^{2 \rho(\log a)} t_{1}^{\operatorname{dim} p_{1}-1} f\left(X^{k a w}\right) d t_{1} d t_{2} \\
& =c_{X} \int_{G / G_{X}} f\left(X^{x}\right) d x
\end{aligned}
$$

and $c_{X}=$ const $/ c_{Z_{0}}$ and $Z_{0}$ depend only on the conjugacy class of $X$ by Proposition 3.1.

This completes Case I.

Case II. ad $H$ has even as well as odd eigenvalues. We can identify $X, H$, $Y, W \in \operatorname{su}(2,1) \subseteq g$ with

$$
\begin{gathered}
X=\left[\begin{array}{rrr}
0 & 0 & 0 \\
0 & i / 2 & i / 2 \\
0 & -i / 2 & -i / 2
\end{array}\right], \quad Y=\left[\begin{array}{rrr}
0 & 0 & 0 \\
0 & +i / 2 & -i / 2 \\
0 & +i / 2 & -i / 2
\end{array}\right], \\
H=\left[\begin{array}{rrr}
0 & 0 & 0 \\
0 & 0 & -1 \\
0 & -1 & 0
\end{array}\right]
\end{gathered}
$$

and

$$
W=\left[\begin{array}{lll}
0 & 0 & 1 \\
0 & 0 & 0 \\
1 & 0 & 0
\end{array}\right]
$$

Then

$$
Z_{0}=\left[\begin{array}{rrr}
0 & 0 & 0 \\
0 & i & 0 \\
0 & 0 & -i
\end{array}\right]
$$

Let $w=\exp t_{1} W$ and $a=\exp t_{2} H$. Then we have the relation

$$
Z_{0}^{w}=i\left[\begin{array}{lll}
\sinh ^{2} t_{1} & 0 & -\sinh t_{1} \cosh t_{1} \\
0 & 1 & 0 \\
\sinh t_{1} \cosh t_{1} & 0 & -\cosh t_{1}
\end{array}\right]
$$

Then

$$
t Z_{0}^{w}=\left[\left(\frac{1+\cosh ^{2} t_{1}}{2}\right)\left(X-t^{2} Y\right)+Z_{t, t_{1}}\right]^{\exp \theta H}
$$

where $\theta=\frac{1}{2} \log t$ and

$$
Z_{t, t_{1}}=-\frac{1}{2} \sinh 2 t_{1}\left(t^{1 / 2} X_{1}+t \tau X_{1}\right)+\frac{1}{2} t \sinh ^{2} t_{1} X_{0}
$$


Here $X_{1} \in g_{1}$ and $X_{0} \in g_{0}$ are given by

$$
X_{1}=\left[\begin{array}{rrr}
0 & i / 2 & i / 2 \\
i / 2 & 0 & 0 \\
-i / 2 & 0 & 0
\end{array}\right], \quad X_{0}=\left[\begin{array}{rrr}
2 i & 0 & 0 \\
0 & -i & 0 \\
0 & 0 & -i
\end{array}\right]
$$

From formula (12) we see that $Z_{t, t_{1}} \rightarrow 0$ when $t \rightarrow 0$.

In the notation of Proposition 4.8 we consider

$$
\int_{0}^{\infty} \int_{0}^{\infty} g\left(t_{1}, t_{2}\right) f\left(t Z_{0}^{a w}\right) d t_{1} d t_{2}
$$

By using relations (10) and (11) we make the change of variables $t_{2}^{\prime}=t_{2}+$ $\theta$. The integral in (12) becomes

$$
\int_{0}^{\infty} \int_{(1 / 2) \log t}^{\infty} g\left(t_{1}, t_{2}-\theta\right) f\left(t, t_{1}, t_{2}\right) d t_{1} d t_{2}
$$

where

$$
f\left(t, t_{1}, t_{2}\right)=f\left[\left(\frac{1+\cosh ^{2} t_{1}}{2}\left(X-t^{2} Y\right)+Z_{t, t_{1}}\right)^{a}\right] .
$$

LEMMA 6.4. The function $f\left(t, t_{1}, t_{2}\right)$ has compact support in $t_{1}$ and $t_{2} \geqslant 0$ independent of $t$.

Proof. Since $X$ is orthogonal to $Y$ and $Z_{t, t_{1}}$ we have the relation

$$
\left\|\left\{\left(\frac{1+\cosh ^{2} t_{1}}{2}\right)\left(X-t^{2} Y\right)+Z_{t, t_{1}}\right\}^{\exp t_{2} H}\right\|^{2} \geqslant\left(\frac{1+\cosh ^{2} t_{1}}{2}\right)^{2} e^{4 t_{2}}\|X\|^{2} .
$$

The statement in the lemma now follows from inequality (16) and the fact that $f$ has compact support.

We rewrite the integral in (14) as

$$
\begin{aligned}
\int_{0}^{\infty} \int_{(1 / 2) \log t}^{0} g\left(t_{1}, t_{2}-\theta\right) f\left(t, t_{1}, t_{2}\right) d t_{1} d t_{2} \\
+\int_{0}^{\infty} \int_{0}^{\infty} g\left(t_{1}, t_{2}-\theta\right) f\left(t, t_{1}, t_{2}\right) d t_{1} d t_{2}
\end{aligned}
$$

Lemma 6.5. Let $r=\operatorname{dim}^{C^{2} t_{\mathrm{g}}} X$ and $n=\operatorname{dim} \mathrm{g}$. Then

$$
2 \rho(H)=n-r \text {. }
$$


Proof. The lemma is an immediate consequence of the relations

$$
\begin{gathered}
2 \rho(H)=\operatorname{dim} g_{1}+2 \operatorname{dim} g_{2}, \\
n=\operatorname{dim} g_{0}+2 \operatorname{dim} g_{1}+2 \operatorname{dim} g_{2}, \\
r=\operatorname{dim} \mathcal{L}+\operatorname{dim} g_{1}+\operatorname{dim} g_{2}, \quad \text { and } \\
\operatorname{dim} g_{0}-\operatorname{dim} \mathcal{L}=\operatorname{dim} g_{2} .
\end{gathered}
$$

Proposition 6.6. Let $f \in C_{c}^{\infty}(g)$. Then

$$
\lim _{t \rightarrow 0^{+}} t^{(n-r) / 2} \int_{G / G_{Z_{0}}} f\left(t Z_{0}^{x}\right) d x=c_{X} \int_{G / G_{X}} f\left(X^{x}\right) d x
$$

and $c_{X}$ depends only on the conjugacy class of $X$ in $g$.

Proof. We note that in formula (17) $g \neq 0$ over the range of the integral as we may take $\pm \operatorname{det} \phi$ in the notation of Proposition 4.8. From formula (11) we conclude that

$$
\lim _{t \rightarrow 0}\left\{\left(\frac{1+\cosh ^{2} t_{1}}{2}\right)\left(X-t^{2} Y\right)+Z_{t, t_{1}}\right\}^{a}=\frac{1+\cosh ^{2} t_{1}}{2} X^{a} .
$$

As in Case I we select a basis of $\mathfrak{f} / \mathscr{L}_{0}$ consisting of vectors $X_{i}+\tau X_{i}$ and $Y_{j}$ where $X_{i} \in g_{i}$ with $i>0$ and $Y_{j} \in \mathcal{L}$ orthogonal to $\mathfrak{L}_{0}$. For $\mathfrak{g}_{Z}$ we select a basis consisting of $Y_{j}$ and $Z_{k}$. Then

$$
\begin{aligned}
& t^{(n-r) / 2} g\left(t_{1}, t_{2}-\theta\right) \\
& =t^{(n-r) / 2} \operatorname{det}\left(e^{-i t_{2} t^{i / 2} X_{i}}+e^{i t_{2}-i / 2} \tau X_{i}, Y_{j}, H, W, \operatorname{Ad} w Y_{j}, \operatorname{Ad} w Z_{k}\right) \\
& =\operatorname{det}\left(e^{-i t_{2}{ }^{i} X_{i}}+e^{i t_{2}} \tau X_{i}, Y_{j}, H, W, \operatorname{Ad} w Y_{j}, \operatorname{Ad} w Z_{k}\right)
\end{aligned}
$$

by multiplying the $t$ inside the determinant. Using formula (18) this expression converges to

$$
e^{2 \rho(\log a)} c\left(t_{1}\right)=e^{2 \rho(\log a)} \operatorname{det}\left(\tau X_{i}, Y_{j}, H, W, \operatorname{Ad} w Y_{j}, \operatorname{Ad} w Z_{k}\right) .
$$

Due to Lemma 6.4 we can use the dominated convergence for the second term in (17). Its limit is

$$
\int_{0}^{\infty} \int_{0}^{\infty} e^{2 \rho(\log a)} c\left(t_{1}\right) f\left(\frac{1+\cosh ^{2} t_{1}}{2} X^{a}\right) d t_{1} d t_{2}
$$

For the first term in (17) we expand in powers of $e^{t_{2}}$. For $0<j \leqslant 2 \rho(H)$ we

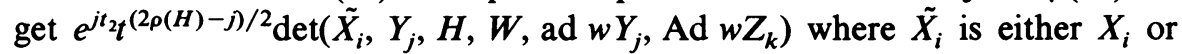
$\tau X_{i}$. Then $e^{j t_{2}} f\left(t, t_{1}, t_{2}\right)$ is bounded by an absolutely integrable function for any $t_{1}$ and $t_{2}$ so we can use the bounded convergence theorem for each term. Only the term for $j=2 \rho(H)$ contributes anything. We get 


$$
\int_{0}^{\infty} \int_{-\infty}^{0} e^{2 \rho(\log a)} c\left(t_{1}\right) f\left(\frac{1+\cosh ^{2} t_{1}}{2} X^{a}\right) d t_{1} d t_{2} .
$$

For $-2 \rho(H) \leqslant j<0$ we make the change of variables $u=t_{2}-\log t$. The integrals transform into

$$
\begin{aligned}
& \int_{0}^{\infty} \int_{-(1 / 2) \log t}^{\infty} e^{j u t(2 \rho(H)+j) / 2} \\
& \cdot \operatorname{det}\left(\tilde{X}_{i}, Y_{j}, H, W, \operatorname{Ad} w Y_{j}, \operatorname{Ad} Z_{k}\right) \tilde{f}\left(t, t_{1}, u\right) d t_{1} d t_{2}
\end{aligned}
$$

where $\tilde{X}_{i}$ is either $X_{i}$ or $\tau X_{i}$ and $\tilde{f}\left(t, t_{1}, u\right)=f\left(t, t_{1}, u+\log t\right)$ is a bounded function in $\left(t, t_{1}, u\right)$. We can apply the bounded convergence theorem. Since $-\frac{1}{2} \log t \rightarrow+\infty$ the limit of any of these integrals is 0 . Remains to consider the case $j=0$. The same argument as in Case I applies. We have obtained the formula

$$
\begin{aligned}
\lim _{t \rightarrow 0^{+}} t^{(n-r) / 2} \int_{0}^{\infty} \int_{0}^{\infty} g\left(t_{1}, t_{2}\right) f\left(t Z_{0}^{a w}\right) d t_{1} d t_{2} \\
\quad=\int_{0}^{\infty} \int_{-\infty}^{\infty} e^{2 \rho(\log a)} c\left(t_{1}\right) f\left(\frac{1+\cosh ^{2} t_{1}}{2} X\right) d t_{1} d t_{2}
\end{aligned}
$$

We make the change of variables $t^{\prime}=t+\frac{1}{2} \log \left(\left(1+\cosh ^{2} t_{1}\right) / 2\right)$ in the right-hand side of (22) to get

$$
\int_{0}^{\infty} \frac{c\left(t_{1}\right)}{\left(\left(1+\cosh ^{2} t_{1}\right) / 2\right)^{\rho(H)}} d t_{1} \int_{-\infty}^{\infty} e^{2 \rho(\log a)} f\left(X^{a}\right) d t_{2}
$$

The proof of the proposition is completed in the same manner as in Case I. We now put these results together in the following theorem.

THEOREM 6.7. Let $f \in C_{c}^{\infty}(G)$. Then

$$
\lim _{t \rightarrow 0^{+}} t^{(n-r) / 2} \int_{G / G_{Z_{0}}} f\left(z_{t}^{x}\right) d x=c_{X} \int_{G / G_{u}} f\left(u^{x}\right) d x
$$

where $u=\exp X, z_{t}=\exp t Z_{0}, r=\operatorname{dim}_{\operatorname{Cent}} X$ and $\{X, H, Y\}$ is an arbitrary Lie triple.

Proof. Let $\theta=\{Z \in g$ : ad $Z$ has eigenvalues $\lambda$ with $|\operatorname{Im} \lambda|<\pi\}$. It is well known that the map exp: $\theta \rightarrow G$ is a diffeomorphism onto an open set $\mathscr{P}$. If $u=\exp X$ and $z_{t}=\exp t Z_{0}$ then $u$ and $z_{t}$ are contained in $\mathscr{P}$ for $t$ small enough. In addition, $\mathcal{O}$ and $\mathcal{P}$ are invariant by the adjoint action so the orbits of $u$ and $z_{t}$ are contained in $\mathscr{P}$. Without loss of generality we may assume that supp $f \subseteq \mathcal{P}$. Let $F=f \circ \exp$. If $F$ does not have compact support we can always find $F_{1}$ with support in a set $\theta_{f}$ such that 
(1) $\theta_{f} \subseteq \vartheta$ and $\operatorname{supp} f \subseteq \exp \theta_{f}$,

(2) $F_{1} \in C_{c}^{\infty}(g)$,

(3) $\left.F_{1}\right|_{O_{f}}=f \circ \exp$.

By applying Propositions 6.3 or 6.6 to $F_{1}$ and $\{X, H, Y\}$ we get

$$
\lim _{t \rightarrow 0^{+}} t^{(n-r) / 2} \int_{G / G_{Z_{0}}} F_{1}\left(t Z_{0}^{x}\right) d x=c_{X} \int_{G / G_{X}} F_{1}\left(X^{x}\right) d x .
$$

We now note that $G_{X}=G_{u}$ and $G_{Z_{0}}=G_{z_{t}}$ for $t$ small enough. The first statement follows from Lemma 5.1. If $t$ is small enough the eigenvalues of $t Z_{0}$ have absolute value less than $\pi$. Then $\operatorname{Cent}_{G_{c}} t Z_{0}=\operatorname{Cent}_{G_{c}} z_{t}$ is a connected group. Thus we can write

$$
\lim _{t \rightarrow 0^{+}} t^{(n-r) / 2} \int_{G / G_{Z_{0}}} f\left(z_{t}^{x}\right) d x=c_{X} \int_{G / G_{u}} f\left(u^{x}\right) d x
$$

and the proof is complete.

We rewrite this formula in terms of the distribution $\Phi_{f}^{B}$ defined in $\$ 1$.

COROLlaRY 6.8. Under the assumptions of Theorem 6.7 let $p=r-s$ where $s=\operatorname{dim}$ Cent $_{g} H$. Then

$$
\left.\left(\frac{d}{d t}\right)^{p}\right|_{t=0} \Phi_{f}^{B}\left(z_{t} ; \bar{\omega}_{Z_{0}}\right)=\Phi_{f}^{B}\left(1 ; \bar{\omega}_{Z_{0}} Z_{0}^{p}\right)=c_{u} \int_{G / G_{u}} f\left(u^{x}\right) d x
$$

Proof. Fix a connected component $B^{+} \subseteq B^{\prime}$ such that $z_{t} \in \operatorname{cl}\left(B^{+}\right)$. By the work of Harish-Chandra it is known that $\Phi_{f}^{B}\left(z_{t} ; \bar{\omega}_{Z_{0}}\right)$ is well defined independent of $B^{+}$. By Lemma 2.7 applied to $\gamma=z_{t}$ we get

$$
\Phi_{f}^{B}\left(z_{t} ; \bar{\omega}_{Z_{0}}\right)=k_{t} \int_{G / G_{Z_{0}}} f\left(z_{t}^{x}\right) d x
$$

where

$$
k_{t}=d_{z_{0}} \xi_{-\rho}\left(z_{t}\right) \prod_{\beta \in P_{g / Z_{0}}}\left(\xi_{\beta}\left(z_{t}\right)-1\right) .
$$

Then

$$
\lim _{t \rightarrow 0^{+}} t^{(n-r) / 2} k_{t}^{-1} \Phi_{f}^{B}\left(z_{t} ; \bar{\omega}_{Z_{0}}\right)=c_{X} \int_{G / G_{u}} f\left(u^{x}\right) d x
$$

by Theorem 6.7. Since $\left[P_{g / Z_{0}}\right]=(n-s) / 2$ we can write

$$
\lim _{t \rightarrow 0^{+}} t^{(n-s) / 2} k_{t}^{-1}=\frac{1}{d_{Z_{0}}} \prod_{\beta \in P_{g / Z_{0}}} \beta\left(Z_{0}\right)^{-1} .
$$

Thus, since $\Phi_{f}^{B}$ is a Schwartz function, we can write

$$
\lim _{t \rightarrow 0^{+}} t^{-p} \Phi_{f}^{B}\left(z_{t} ; \bar{\omega}_{Z_{0}}\right)=\text { const } \int_{G / G_{u}} f\left(u^{x}\right) d x .
$$


The proof will be complete if we show that

$$
\begin{aligned}
\lim _{t \rightarrow 0^{+}}(p !) t^{-p} \Phi_{f}^{B}\left(z_{t} ; \bar{\omega}_{Z_{0}}\right) & =\lim _{t \rightarrow 0^{+}} \Phi_{f}^{B}\left(z_{t} ; \bar{\omega}_{Z_{0}} Z_{0}^{p}\right) \\
=\Phi_{f}^{B}\left(1 ; \bar{\omega}_{Z_{0}} Z_{0}^{p}\right) & =\left.\left(\frac{d}{d t}\right)^{p}\right|_{t=0} \Phi_{f}^{B}\left(z_{t} ; \bar{\omega}_{Z_{0}}\right) .
\end{aligned}
$$

This is a consequence of the elementary Lemma 6.9. Putting all the coefficients together we get the following expression for $c_{u}$.

$$
c_{u}=(p !) d_{Z_{0}} c_{X} \prod_{\beta \in P_{g / Z_{0}}}\left(\beta, Z_{0}\right)
$$

LEMma 6.9. Let $F \in C^{\infty}(0, \varepsilon]$ be such that for any integer $k, D_{t}^{k} F$ has a limit when $t \rightarrow 0 \quad\left(D_{t}=d / d t\right)$. Then if $\lim _{t \rightarrow 0^{+}} t^{-p} F(t)$ exists, it equals $(p !)^{-1} D_{t}^{p} F(0)$.

Proof. Extend $F$ to a $C^{p+2}$ function on $(-\varepsilon, \varepsilon)$. The Taylor series expansion of $F$ is

$$
F(t)=F(0)+\frac{F^{\prime}(0)}{1 !} t+\cdots+\frac{F^{(p)}(0)}{p !} t^{p}+O\left(t^{p+1}\right) .
$$

If $\lim _{t \rightarrow 0} t^{-p} F(t)$ exists, then it is clear that

$$
F(0)=\cdots=F^{(p-1)}(0)=0 \text { and } \lim _{t \rightarrow 0^{+}} t^{-p} F(t)=(p !)^{-1} D_{t}^{p} F(0) .
$$

7. The Fourier transform of the unipotent integral. In this section we calculate the Fourier transform of the distribution $T_{u}(f)$ for $u \in G$. This will be done by using the results in $\$ 6$ and [13].

We use the notation in $\$ 2$. In order to use the results in [12] we explain the relevant notation used there. Let $B$ be the compact CSG. Then $A_{K}=$ $Z(A) A_{K}^{0}$ where $A_{K}^{0}$ is the connected component of $A_{K}$ and $Z(A)=\{1, v\}$ where $v=\exp \left(\sqrt{-1} \pi H_{\alpha}\right)=\exp \left(\pi\left(E_{\alpha}-E_{-\alpha}\right)\right)$.

We let $\mathfrak{b}_{2}=\mathbf{R}\left(E_{\alpha}-E_{-\alpha}\right)$ and $\mathfrak{b}_{1}$ its orthogonal complement in $\mathfrak{b}$. Let $B_{1}$ and $B_{2}$ be the corresponding subgroups. Let now $Z \in \mathfrak{b}$ and $z=\exp Z$. Set $P_{Z}$ as in $\S 2$, Lemma 2.7. Let $W_{z}(G, B)$ be the subgroup of $W(G, B)$ generated by the compact roots in $P_{Z}$. Choose $w_{1}=1, \ldots, w_{n}$ such that

$$
W(G, B)=\bigcup_{i=1}^{N} W_{z}(G, B) w_{i} \quad \text { (disjoint union). }
$$

Then if $w \in W(G, B), w=w_{2} w_{i}$. Moreover

$$
w_{i}^{-1} z=z_{1}\left(w_{i}\right) z_{2}\left(w_{i}\right), \quad z_{1} \in B_{1}, z_{2} \in B_{2} .
$$

Since this decomposition is unique only up to $\{1, v\}$ we normalize $z_{2}$ such that

$$
z_{2}\left(w_{i}\right)=\exp \theta_{w_{i}}\left(E_{\alpha}-E_{-\alpha}\right), \quad-\pi / 2 \leqslant \theta_{w_{i}}<\pi / 2
$$


We will take $Z=t Z_{0}$ for $t$ small enough so that $P_{Z}$ and $P_{z}$ are identical.

We define the functions

$$
\begin{aligned}
& F_{\chi}^{ \pm}(w: \nu: z)=\overline{w \chi(z)}\left\{e^{\mp \nu \pi / 2}\left(w \xi_{\alpha}(z)\right)^{\sqrt{-1} \nu / 2}-e^{ \pm \nu \pi / 2}\left(w \xi_{\alpha}(z)\right)^{-\sqrt{-1} \nu / 2}\right\} \\
& =\left\{e^{\mp \nu \pi / 2} \exp \left[\left(\log \bar{\chi}+\frac{\sqrt{-1} \nu}{2} \tilde{\alpha}, w^{-1} Z\right)\right]\right. \\
& \left.-e^{ \pm \nu \pi / 2} \exp \left[\left(\log \bar{\chi}-\frac{\sqrt{-1} \nu}{2} \tilde{\alpha}, w^{-1} Z\right)\right]\right\}, \\
& G_{\chi}^{ \pm}(w: \nu: z)=\overline{ \pm w \chi(z)}\left\{e^{\mp v \pi / 2}\left(w \xi_{\tilde{\alpha}}(z)\right)^{\sqrt{-1} \nu / 2}\right. \\
& \left.+e^{ \pm \nu \pi / 2}\left(w \xi_{\dot{\alpha}}(z)\right)^{-\sqrt{-1} \nu / 2}\right\} \\
& = \pm\left\{e^{\mp \nu \pi / 2}\left(\log \bar{\chi}+\frac{\sqrt{-1} \nu}{2} \tilde{\alpha}, w^{-1} Z\right)\right. \\
& \left.+e^{ \pm \nu \pi / 2}\left(\log \bar{\chi}-\frac{\sqrt{-1} \nu}{2} \tilde{\alpha}, w^{-1} Z\right)\right\} \text {. }
\end{aligned}
$$

Let $r=[P], r_{z}=\left[P_{z}\right]$ and $r_{I}=\left[P_{I}\right]$. We also define $\hat{A}_{\bar{K}}^{ \pm}=\{\chi: \chi(v)= \pm 1\}$ and $\varepsilon(\chi)=\operatorname{sgn} \prod_{\alpha \in P_{I}}(\log \chi, \alpha)$.

We are now ready to state the main result of this section.

Theorem 7.1. Let $u=\exp X$ be unipotent. Then the distribution $c_{u} T_{u}(f)$ equals

$$
\begin{aligned}
& (-1)^{r+z_{0}} \sum_{\mu \in L_{B}}\left|\prod_{\beta \in P_{Z_{0}}}(\mu, \beta)\right|\left[\overline{\left(\mu, Z_{0}\right)}\right]^{p} \Theta_{\mu}(f) \\
& +(\sqrt{-1} / 4)(-1)^{r_{1}}\left[W_{Z_{0}}(G, B)\right] /[W(G, A)] \sum_{\chi \in \hat{A}_{\bar{K}}} \varepsilon(\chi) \\
& \times\left\{\sum_{\substack{w_{i} \\
0<\theta_{w_{i}}<\pi / 2}} \operatorname{det}\left(w_{i}\right) \int_{-\infty}^{+\infty} T^{(x, \nu)}(f)\left[F_{x}^{+}\left(w_{i}: \nu: 1 ; \bar{\omega}_{Z_{0}} Z_{0}^{p}\right) / \sinh (\nu \pi / 2)\right] d \nu\right. \\
& \left.+\sum_{-\pi / 2<\theta_{w_{i}}<0} \operatorname{det}\left(w_{i}\right) \int_{-\infty}^{+\infty} T^{(x, \nu)}(f)\left[F_{x}^{-}\left(w_{i}: \nu: 1 ; \bar{\omega}_{Z_{0}} Z_{0}^{p}\right) / \sinh (\nu \pi / 2)\right] d \nu\right\} \\
& +(\sqrt{-1} / 4)(-1)^{r_{r}}\left[W_{Z_{0}}(G ; B)\right] /[W(G, A)] \sum_{\chi \in \hat{A}_{\bar{K}}} \varepsilon(\chi) \\
& \times\left\{\sum_{\substack{w_{i} \\
0<\theta_{w_{i}}<\pi / 2}} \operatorname{det}\left(w_{i}\right) \int_{-\infty}^{+\infty} T^{(\chi, \nu)}(f)\left[G_{x}^{+}\left(w_{i}: \nu: 1 ; \bar{\omega}_{Z_{0}} Z p\right) / \cosh (\nu \pi / 2)\right] d \nu\right. \\
& \left.+\sum_{\substack{w_{i} \\
-\pi / 2<\theta_{w_{i}}<0}} \operatorname{det}\left(w_{i}\right) \int_{-\infty}^{+\infty} T^{(\chi, \nu)}(f)\left[G_{\chi}^{-}\left(\ddot{w}_{i}: \nu: 1 ; \bar{\omega}_{Z_{0}} Z_{0}^{p}\right) / \cosh (\nu \pi / 2)\right] d \nu\right\} .
\end{aligned}
$$


Proof. This formula follows immediately by applying Theorem 6.7 to Theorems 3.19 and 5.21 in [13], substituting $y=z_{t}=\exp t Z_{0}$ and differentiating. Lemma 68 on p. 315 in [5c] for the discrete series part and the form of $T^{(\chi, \nu)}$ given in [13] can be used to justify term by term differentiation.

The formulas for $F_{\chi}^{ \pm}$and $G_{\chi}^{ \pm}$occurring in the statement are given below

$$
\begin{aligned}
& F_{\chi}^{ \pm}\left(w: \nu: 1 ; \bar{\omega}_{Z_{0}} Z_{0}^{p}\right)=\left\{c_{1} e^{\mp \nu \pi / 2}\left[\left(\overline{\log \chi}+\frac{\sqrt{-1} \nu}{2} \tilde{\alpha}, w^{-1} Z_{0}\right)\right]^{p}\right. \\
&\left.-c_{2} e^{ \pm \nu \pi / 2}\left[\left(\log \bar{\chi}-\frac{\sqrt{-1} \nu}{2} \tilde{\alpha}, w^{-1} Z_{0}\right)\right]^{p}\right\} \\
& G_{\chi}^{ \pm}\left(w: \nu: 1 ; \bar{\omega}_{Z_{0}} Z_{0}^{p}\right)=\left\{c_{1} e^{\mp \nu \pi / 2}\left[\left(\log \bar{\chi}+\frac{\sqrt{-1} \nu}{2} \tilde{\alpha}, w^{-1} Z_{0}\right)\right]^{p}\right. \\
&\left.+c_{2} e^{ \pm \nu \pi / 2}\left[\left(\log \bar{\chi}-\frac{\sqrt{-1} \nu}{2} \tilde{\alpha}, w^{-1} Z_{0}\right)\right]^{p}\right\},
\end{aligned}
$$

where

$$
\begin{aligned}
& c_{1}=\prod_{\beta \in P_{Z_{0}}}\left(w\left(\log \bar{\chi}+\frac{\sqrt{-1} \nu}{2} \tilde{\alpha}\right), \beta\right), \\
& c_{2}=\prod_{\beta \in P_{Z_{0}}}\left(w\left(\log \bar{\chi}-\frac{\sqrt{-1} \nu}{2} \tilde{\alpha}\right), \beta\right) .
\end{aligned}
$$

REMARK. These formulas become simpler when $Z_{0}$ is regular, i.e. $\mathfrak{g}=$ $\mathrm{sl}(2, \mathbf{R})$ or $g=\operatorname{su}(2,1)$ as Theorem 3.19 in [13] suggests. Similar formulas for $u \in G$ arbitrary were calculated in [2] using Jordan decomposition.

8. The constant $c_{u}$. In this section we evaluate the constant $c_{u}$ relevant to the calculations in [10]. In view of this we note that if the discrete series part of the distribution $T_{u}(f)$ is $\Sigma a(\mu) \theta_{\mu}(f)$ then up to a constant factor,

$$
a(\mu)=\left(\overline{\mu, Z_{0}}\right)^{p} \prod_{\beta \in P_{Z_{0}}}(\mu, \beta) / \prod_{\beta \in P_{g / Z_{0}}}\left(\beta, Z_{0}\right) .
$$

Lemma 8.1. Suppose that there is an element $x \in G$ such that $X^{x}=-X$. Then the discrete series part in Theorem 7.1 is equal to zero.

Proof. If $X^{x}=-X$ then there is $w \in W(G, B)$ such that $Z_{0}^{w}=-Z_{0}$ by the results in \$3. Furthermore we must have $\operatorname{dim} g_{2}>1$. By the formulas for $\theta_{\mu}$ in [12] we know that $\theta_{w \mu}=\varepsilon(w) \theta_{\mu}$. Both $\theta_{\mu}$ and $\theta_{w \mu}$ occur in the sum. We 
compute $a(w \mu)$. We have

$$
\begin{aligned}
a(w \mu) & =\left(\overline{w \mu, Z_{0}}\right)^{p} \prod_{\beta \in P_{Z_{0}}}(w \mu, \beta) / \prod_{\beta \in P_{g / Z_{0}}}\left(\beta, Z_{0}\right) \\
& =(-1)^{p} \varepsilon_{Z_{0}}(w)\left(\overline{\mu, Z_{0}}\right)^{p} \prod_{\beta \in P_{Z_{0}}}(\mu, \beta) / \prod_{\beta \in P_{g / Z_{0}}}\left(\beta, Z_{0}\right) \\
& =(-1)^{p} \varepsilon_{Z_{0}}(w) a(\mu)
\end{aligned}
$$

where $\varepsilon_{Z_{0}}(w)$ is the number of roots in $P_{Z_{0}}$ mapped into $-P_{Z_{0}}$ by $w$. We recall that $p=(r-s) / 2$ also equals $\frac{1}{2} \operatorname{dim} g_{1}$. Assume $p>0$. We will show that $p$ is even or equivalently that $\operatorname{dim} g_{1} \equiv 0(\bmod 4)$. We recall that the complexification of $g_{1}$ is generated by root vectors of roots $\alpha_{1}$ such that $\alpha_{1}(H)=$ 1. Then $\sigma \alpha_{1} \neq \alpha_{1}$ and $\sigma \alpha_{1}(H)=1$. Since $\operatorname{dim} g_{2}>1$ let $\beta \in P$ be a root such that $\beta \neq \alpha$ and $\beta(H)=2$. By Lemma 2.4 either $\beta-\alpha_{1}$ or $\beta-\sigma \alpha_{1}$ is a root, say $\beta-\alpha_{1}=\beta_{1}$ is a root. Clearly $\beta-\sigma \alpha_{1}$ cannot also be a root since otherwise either $\left(\beta-\alpha_{1}\right)-\left(\beta-\sigma \alpha_{1}\right)=\sigma \alpha_{1}-\alpha_{1}$ or $\left(\beta-\alpha_{1}\right)-\sigma(\beta-$ $\left.\sigma \alpha_{1}\right)=\beta-\sigma \beta$ would be a root. Thus $P_{1}$ splits into disjoint sets of the form $\left\{\alpha_{1}, \sigma \alpha_{1}, \beta_{1}, \sigma \beta_{1}\right\}$. Thus $\left[P_{1}\right] \equiv 0(\bmod 4)$. Therefore $a(w \mu)=\varepsilon_{Z_{0}}(w) a(\mu)$. Finally we show that $\varepsilon_{Z_{0}}(w) \varepsilon(w)=-1$. We may assume that $x$ is such that $X^{x}=-X, Y^{x}=-Y$ and $H^{x}=H$. Let $\mathrm{m}=\operatorname{Cent}_{K} H$ and $\mathrm{m}_{z_{0}}=$ Cent $_{\mathrm{m}} Z_{0}$. Then $\mathfrak{m}_{z_{0}}^{x}=\mathfrak{m}_{Z_{0}}$ is a compact subalgebra containing $a_{K}$ as a Cartan subalgebra. Thus we may replace $x$ with $w \in W(G, B)$ with the same properties. In addition, we may consider $w$ as an element of $W(G, J)$ as well. But $w$ maps $Z_{0}$ into $-Z_{0}$ so it maps $P_{g / Z_{0}}$ into $-P_{g / Z_{0}}$. Since $\left[P_{g / Z_{0}}\right]=\operatorname{dim} g_{1}+\operatorname{dim} g_{2}$ is odd and the complement of $P_{g / Z_{0}}$ in $P$ is $P_{Z_{0}}$ we have $\varepsilon(w)=(-1) \varepsilon_{Z_{0}}(w)$. This completes the proof of the lemma.

Thus the only cases when the unipotent orbit has a nontrivial discrete series contribution is when $\operatorname{dim} g_{2}=1$. Then $\operatorname{dim} g_{4}=0$ and the only cases are the groups $\operatorname{su}(n, 1)$ for $n \geqslant 1$. The case $n=1$ is well known. Thus we assume $\operatorname{dim} g_{1}>0$. This is Case II in $\S 6$. Let $w=\exp t_{1} W$ and $X, H, Y, W$ be as in that case. From the proof of Proposition 6.6 we have to calculate $c\left(t_{1}\right)$ defined by (20). Let $Z_{1} \in g^{-} \oplus \mathcal{L} / \varrho_{0}$ where $g^{-}=\Sigma_{i<0} g^{i}$ and $Z_{2} \in g_{Z}$. Then we have to evaluate the determinant of $\phi: g^{-} \oplus \mathcal{L} / \mathcal{L}_{0} \times \mathbf{R}^{2} \times g_{Z} \rightarrow g$ given by $\phi\left(Z_{1}, s_{2}, s_{1}, Z_{2}\right)=Z_{1}^{a^{-1}}+s_{2} H+s_{1} W+Z_{2}^{w}$. By choosing a basis as in Case II we get $e^{2 \rho(\log a)} \operatorname{det}\left(\tau X_{i}, Y_{j}, H, W, \operatorname{Ad} w Y_{j}, \operatorname{Ad} w Z_{k}\right)$. We note that $\mathrm{g}_{Z}=\mathcal{L}$ $+\mathbf{R} Z_{0}$ in this case. Also $W$ and $H$ are conjugate in $\operatorname{su}(2,1)$ by some element $x=s_{\tilde{\alpha}_{1}}$ where $s_{\tilde{\alpha}_{1}}$ is the reflection in $W(G, B)$ corresponding to a simple root $\alpha_{1} \in \Delta\left(g_{c}, j_{c}\right)$. Suppose $E_{\beta_{1}-\alpha_{1}} \in \mathcal{L}_{c}$ is a root vector. Then

$$
w E_{\beta_{1}-\alpha_{1}}=w E_{\tilde{\beta}_{1}-\tilde{\alpha}_{1}}=s_{\tilde{\alpha}_{1}} \exp t_{1} H s_{\tilde{\alpha}_{1}} E_{\tilde{\beta}_{1}-\tilde{\alpha}_{1}}=s_{\alpha_{1}} \exp t_{1} H E_{\tilde{\beta}_{1}} .
$$

Thus it is enough to calculate $\exp t_{1} H E_{\tilde{\alpha}_{1}}$ since $\tilde{\alpha}_{1}$ and $\tilde{\beta}_{1}$ generate a subalgebra isomorphic to $\operatorname{su}(2,1)$. We get $\cosh t_{1} E_{\tilde{\beta}_{1}}-\sinh t_{1} E_{\sigma \tilde{\beta}_{1}}$ and $s_{\tilde{\alpha}_{1}}$ maps 
them into

$$
\cosh t_{1} E_{\tilde{\beta}_{1}-\tilde{\alpha}_{1}}-\sinh t_{1} E_{\tilde{\beta}_{1}}=\cosh t_{1} E_{\beta_{1}-\alpha_{1}}-\sinh t_{1}\left(E_{\beta_{1}} \pm E_{-\sigma \beta_{1}}\right) .
$$

Furthermore, by relations (10) and (11),

$$
Z_{0}^{w}=\frac{1+\cosh ^{2} t_{1}}{2} Z_{0}-\frac{1}{2} \sinh 2 t_{1}\left(X_{1}+\tau X_{1}\right)+\frac{1}{2} \sinh ^{2} t_{1} X_{0}
$$

We write

$$
Z_{0}^{w}=\frac{1+\cosh ^{2} t_{1}}{2} Z_{0}+Z_{t_{1}}
$$

Substituting into the expression for $\varphi$ we get

$$
\begin{aligned}
e^{2 \rho(\log a)} \operatorname{det}\left(\tau X_{i}, Y_{j}, H, W, \text { Ad } w a_{k}, \cosh t_{1} E_{\beta_{1}-\alpha_{1}}\right. & \\
& \left.-\sinh t_{1}\left(E_{\beta_{1}} \pm E_{-\sigma \beta_{1}}\right), Z_{0}^{w}\right) .
\end{aligned}
$$

By elementary column operations this reduces to

$$
2^{p-1} e^{2 \rho(\log a)}\left(\sinh t_{1}\right)^{2 p-2} \operatorname{det}_{\mathrm{su}(2,1)}
$$

where $\operatorname{det}_{\mathrm{su}(2,1)}$ is the corresponding determinant for $g=\mathrm{su}(2,1)$. But this can be calculated explicitly and equals $\sinh 2 t_{1}$. Thus $c\left(t_{1}\right)=\left(\sinh t_{1}\right)^{2 p-2} \sinh 2 t_{1}$ and the integral to be evaluated is

$$
2^{p} \int_{0}^{\infty} \frac{\left(\sinh t_{1}\right)^{2 p-2} \sinh 2 t_{1}}{\left(\left(1+\cosh ^{2} t_{1}\right) / 2\right)^{\rho(H)}} d t_{1}
$$

which is finite since $2 p+2=2 \rho(H)$. We get by setting $u=\sinh t_{1}$

$$
\begin{gathered}
\int_{0}^{\infty} \frac{\left(\sinh t_{1}\right)^{2 p-1} \cosh t_{1}}{\left(1+\frac{\sinh ^{2} t_{1}}{2}\right)^{p+1}} d t_{1}=\int_{0}^{\infty} \frac{u^{2 p-1}}{\left(1+\frac{u^{2}}{2}\right)^{p+1}} d u \\
=2^{p-1} \int_{1}^{\infty} \frac{(v-1)^{p-1}}{v^{p+1}} d v=2^{p-1}(-1)^{p-1} \int_{0}^{1}(1-v)^{p-1} v^{4} d v \\
=2^{p-1}(-1)^{p-1} B(p-1,4)
\end{gathered}
$$

where $v=1+u^{2} / 2$ in the third equality. Putting these calculations together we get the following proposition.

Proposition 8.2. In the case when the discrete series contribution of $T_{u}$ is not zero, the constant $c_{u}$ can be expressed as

$$
c_{u}=2^{2 p-1}(-1)^{p-1} B(p-1,4) \cdot c_{Z_{0}}^{-1} \cdot d_{Z_{0}} \cdot(p !) \prod_{\beta \in P_{g / Z_{0}}}\left(\beta, Z_{0}\right)
$$


where $c_{Z_{0}}$ is defined in Proposition 4.8 and $d_{Z_{0}}$ in Lemma 2.7.

Proof. The statement follows easily from the previous calculation and Corollary 6.8 .

\section{REFERENCES}

1. S. Araki, On root structure and an infinitesimal classification of irreducible symmetric spaces, J. Math. Osaka Univ. 13 (1962), 1-34.

2. D. Barbasch, Fourier inversion formulas of orbital integrals, thesis, University of Illinois at Urbana-Champaign, 1976.

3. A. Borel, Seminar on algebraic groups and related finite groups, Lecture Notes in Math., vol. 131, Springer-Verlag, Berlin and New York, 1970.

4a. Harish-Chandra, A formula for semisimple Lie groups, Amer. J. Math. 79 (1957), 733-760.

4b. __ Invariant distributions on Lie algebras, Amer. J. Math. 86 (1964), 271-309.

4c. __ Discrete series for semisimple Lie groups. I, Acta Math. 113 (1965), 241-318.

4d. __, Discrete series for semisimple Lie groups. II, Acta Math. 116 (1966), 1-111.

4e. __ Two theorems on semisimple Lie groups, Ann. of Math. (2) 83 (1966), 74-128.

5. S. Helgason, Differential geometry and symmetric spaces, Academic Press, New York, 1962.

6. B. Herb, Fourier inversion of invariant integrals on semisimple real Lie groups (preprint).

7. B. Herb and P. Sally, Singular invariant eigendistributions as characters, Bull. Amer. Math. Soc. 83 (1977), 252-254.

8a. B. Kostant, The principal three-dimensional subgroup and the Betti numbers of a complex simple Lie group, Amer. J. Math. 81 (1959), 973-1032.

8b. B. Kostant and S. Rallis, On orbits associated with symmetric spaces, Bull. Amer. Math. Soc. 75 (1969), 884-887.

9. G. Mostow, Some new decomposition theorems for semisimple Lie groups, Mem. Amer. Math. Soc., no. 14 (1955), 31-54.

10. S. Osborne and G. Warner, Multiplicities of the integrable discrete series: The case of a non-uniform lattice in an R-rank one semi-simple group (preprint).

11a. R. Rao, Results on even nilpotents, unpublished.

11b. __ Orbital integrals in reductive Lie groups, Ann. of Math. 96 (1972), 505-510.

12. P. Sally, Jr. and G. Warner, The Fourier transform on semisimple Lie groups of real rank one, Acta Math. 131 (1973), 1-26.

13. H. Samelson, Notes on Lie algebras, Van Nostrand, Princeton, N. J., 1969.

14. N. Wallach, Harmonic analysis on homogeneous spaces, Dekker, New York, 1973.

15a. G. Warner, Harmonic analysis on semisimple Lie groups, vols. 1 and 2, Springer-Verlag, Berlin and New York, 1972.

$15 b$. The Selberg trace formula for real rank one (preprint).

Department of Mathematics, Massachusetts institute of Technology, Cambridge, MASSACHUSETTS 02139

Current address: School of Mathematics, Institute for Advanced Study, Princeton, New Jersey 08540 